\title{
On the stochastic Dullin-Gottwald-Holm equation: global existence and wave-breaking phenomena
}

\author{
Christian Rohde and Hao Tang(e)
}

\begin{abstract}
We consider a class of stochastic evolution equations that include in particular the stochastic Camassa-Holm equation. For the initial value problem on a torus, we first establish the local existence and uniqueness of pathwise solutions in the Sobolev spaces $H^{s}$ with $s>3 / 2$. Then we show that strong enough nonlinear noise can prevent blow-up almost surely. To analyze the effects of weaker noise, we consider a linearly multiplicative noise with non-autonomous pre-factor. Then, we formulate precise conditions on the initial data that lead to global existence of strong solutions or to blow-up. The blow-up occurs as wave breaking. For blow-up with positive probability, we derive lower bounds for these probabilities. Finally, the blow-up rate of these solutions is precisely analyzed. Mathematics Subject Classification. Primary: 60H15, 35Q51; Secondary: 35A01, 35B44.
\end{abstract}

Keywords. Stochastic Dullin-Gottwald-Holm equation, Pathwise solution, Blow-up scenario, Global existence, Wave breaking, Blow-up rate.

\section{Contents}

1. Introduction 2

2. Definitions, assumptions and main results 5

2.1. Definitions of the solutions 6

2.2. Assumptions

2.3. Main results and remarks 8

3. Preliminary results 12

4. Proof of Theorem 2.1 14

C. Rohde acknowledges support by Deutsche Forschungsgemeinschaft (DFG, German Research Foundation) under Germany's Excellence Strategy-EXC 2075-39074001. H. Tang is supported by a postdoctoral scholarship of the Alexander von Humboldt Foundation. 
5. Proof of Theorem 2.2: strong nonlinear noise 17

6. Proofs of Theorems 2.3-2.7: non-autonomous linear noise case 21

6.1. Theorem 2.3: global existence for weak noise I 22

6.2. Theorem 2.4: global existence for weak noise II 24

6.3. Theorem 2.5: blow-up scenario 26

6.4. Theorem 2.6: wave breaking and its probability 26

6.5. Theorem 2.7: wave breaking rate 29

$\begin{array}{ll}\text { Acknowledgements } & 30\end{array}$

$\begin{array}{ll}\text { References } & 30\end{array}$

\section{Introduction}

The Dullin-Gottwald-Holm (DGH) equation is a third-order dispersive evolution equation given by

$$
\begin{aligned}
u_{t} & -\alpha^{2} u_{x x t}+c_{0} u_{x}+3 u u_{x}+\gamma u_{x x x} \\
& =\alpha^{2}\left(2 u_{x} u_{x x}+u u_{x x x}\right) \quad \text { in } \quad(0, \infty) \times \mathbb{R} .
\end{aligned}
$$

It was derived by Dullin et al. in [20] as a model governing planar solutions to Euler's equations in the shallow-water regime. The unknown $u=u(t, x)$ in (1.1) stands for the longitudinal velocity component and $\alpha^{2}, \gamma$ and $c_{0}$ are some physical parameters.

The DGH equation (1.1) embeds two different integrable soliton equations. When $\alpha=0,(1.1)$ reduces to the Korteweg-de-Vries (KdV) equation

$$
u_{t}+c_{0} u_{x}+3 u u_{x}+\gamma u_{x x x}=0,
$$

while (1.1) equals to the following Camassa-Holm $(\mathrm{CH})$ equation for the choices $\gamma=0$ and $\alpha=1$,

$$
u_{t}-u_{x x t}+c_{0} u_{x}+3 u u_{x}=2 u_{x} u_{x x}+u u_{x x x} .
$$

Both (1.2) and (1.3) have been studied widely in the literature. We notice that the $\mathrm{CH}$ equation exhibits two interesting phenomenon, namely (peaked) soliton interaction and wave breaking (the solution remains bounded but its slope becomes unbounded in finite time, cf. [12]), while the KdV equation does not model breaking waves [35] (when $c_{0}=0,(1.2)$ admits a smooth soliton). For the $\mathrm{CH}$ equation, wave breaking and the necessary and sufficient criterion for the occurrence of breaking waves in the Cauchy problem with smooth initial data have been analyzed $[10,12,13,43]$. As pointed out in $[11,14,15]$, the essential feature of the $\mathrm{CH}$ equation is the occurrence of traveling waves with a peak at their crest, exactly like that the governing equations for water waves admit the so-called Stokes waves of the greatest height. Bressan\&Constantin $[5,6]$ developed a new approach to the analysis of the $\mathrm{CH}$ equation, and proved the existence of a global conservative and dissipative solutions. Later, 
Holden\&Raynaud [31,32] also obtained global conservative and dissipative solutions using a Lagrangian point of view.

Combining the linear dispersion of the $\mathrm{KdV}$ equation with the nonlocal dispersion of the $\mathrm{CH}$ equation, the DGH equation (1.1) preserves its bi-Hamiltonian structure, is completely integrable (via the inverse scattering transform method [20]) and admits also soliton solutions.

Here, we are interested in stochastic variants of the DGH equation to model energy consuming/exchanging mechanisms in (1.1) that are driven by external stochastic influences. Adding multiplicative noise has also been connected to the prevailing hypotheses that the onset of turbulence in fluid models involves randomness, cf. [7,21,39]. Precisely, our stochastic evolution equation is rewritten as

$$
\begin{aligned}
u_{t} & -\alpha^{2} u_{x x t}+c_{0} u_{x}+3 u u_{x}+\gamma u_{x x x}-\dot{W}\left(1-\alpha^{2} \partial_{x x}^{2}\right) h(t, u) \\
& =\alpha^{2}\left(2 u_{x} u_{x x}+u u_{x x x}\right),
\end{aligned}
$$

where $W$ is a standard 1-D Brownian motion and $h=(t, u)$ is a typically nonlinear function. We notice that the deterministic counterpart of (1.4) is the weakly dissipative $\mathrm{CH}$ equation

$$
u_{t}-u_{x x t}+3 u u_{x}+\lambda\left(1-\partial_{x x}^{2}\right) h(t, u)=2 u_{x} u_{x x}+u u_{x x x}, \quad \lambda>0 .
$$

Equation (1.5) has been introduced and studied for $h(t, u)=u$ in [40,53], In (1.5), the operator $\lambda\left(1-\partial_{x x}^{2}\right)$ is linear and only models the (weak) energy dissipation. In order to model more general random energy exchanges, we consider the possibly nonlinear noise term $-\dot{W}\left(1-\alpha^{2} \partial_{x x}^{2}\right) h(t, u)$ in (1.4).

To compare our model with deterministic weakly dissipative $\mathrm{CH}$ type equations (see $[40,52,53]$ and the references therein), we focus our attention on the case that $\alpha \neq 0$. For convenience, we assume $\alpha=1$ in this paper. When $\alpha=1$, applying the operator $\left(1-\partial_{x x}^{2}\right)^{-1}$ to (1.4) gives rise to the following nonlocal equation

$$
\mathrm{d} u+\left[(u-\gamma) \partial_{x} u+\left(1-\partial_{x x}^{2}\right)^{-1} \partial_{x}\left(u^{2}+\frac{1}{2} u_{x}^{2}+\left(c_{0}+\gamma\right) u\right)\right] \mathrm{d} t=h(t, u) \mathrm{d} W
$$

In (1.6), the operator $\left(1-\partial_{x x}^{2}\right)^{-1}$ in torus $\mathbb{T}=\mathbb{R} / 2 \pi \mathbb{Z}$ is understood as

$$
\left\{\begin{aligned}
{\left[\left(1-\partial_{x x}^{2}\right)^{-1} f\right](x) } & =\left[G_{\mathbb{T}} * f\right](x), \quad \forall f \in L^{2}(\mathbb{T}), \\
G_{\mathbb{T}} & =\frac{\cosh \left(x-2 \pi\left[\frac{x}{2 \pi}\right]-\pi\right)}{2 \sinh (\pi)},
\end{aligned}\right.
$$

where $[x]$ stands for the integer part of $x$. Here we remark that for additive noise, (1.6) has been studied in [42]. In this paper we will consider a more general context with noise driven by a cylindrical Wiener process $\mathcal{W}$, rather than a standard Brownian motion $W$. It is assumed that $\mathcal{W}$ is defined on an auxiliary Hilbert space $U$ which is adapted to a right-continuous filtration $\left\{\mathcal{F}_{t}\right\}_{t \geq 0}$, see Sect. 2 for more details.

With the above notations, the first goal of the present paper is to analyze the existence and uniqueness of pathwise solutions and to determine possible 
blow-up criterion for the periodic boundary value problem

$$
\left\{\begin{array}{l}
\mathrm{d} u+\left[(u-\gamma) \partial_{x} u+F(u)\right] \mathrm{d} t=h(t, u) \mathrm{d} \mathcal{W}, \quad x \in \mathbb{T}=\mathbb{R} / 2 \pi \mathbb{Z}, t>0 \\
u(\omega, 0, x)=u_{0}(\omega, x), \quad x \in \mathbb{T}
\end{array}\right.
$$

where $F(u)=F_{1}(u)+F_{2}(u)+F_{3}(u)$ and

$$
\left\{\begin{array}{l}
F_{1}(u)=\left(1-\partial_{x x}^{2}\right)^{-1} \partial_{x}\left(u^{2}\right), \\
F_{2}(u)=\left(1-\partial_{x x}^{2}\right)^{-1} \partial_{x}\left(\frac{1}{2} u_{x}^{2}\right), \\
F_{3}(u)=\left(1-\partial_{x x}^{2}\right)^{-1} \partial_{x}\left(\left(c_{0}+\gamma\right) u\right) .
\end{array}\right.
$$

Under generic assumptions on $h(t, u)$, we will show that (1.8) has a local unique pathwise solution (see Theorem 2.1 below). Here we remark that Chen et al. in [9] have considered the stochastic $\mathrm{CH}$ equation with additive noise. For the linear multiplicative noise case, we refer to [48] for the stochastic $\mathrm{CH}$ equation, and to [8] for a stochastic modified $\mathrm{CH}$ equation.

For stochastic nonlinear evolution equations, the noise effect is a crucial question to study. Can the noise prevent blow-up or does it even drive the formation of singularities? For example, it is known that the well-posedness of linear stochastic transport equations with noise can be established under weaker hypotheses than its deterministic counterpart (cf. [22,24]). For stochastic scalar conservation laws, noise on the flux may bring some regularization effects [27], and it may also trigger the discrete entropy dissipation in the numerical schemes for conservation laws such that the schemes enjoy some stability properties not present in the deterministic case [37]. Moreover, we refer to $[29,36,46,48]$ for the dissipation of energy caused by the linear multiplicative noise.

However, most existing results on the regularization effects by noise for transport type equations are for linear equations or restricted to linear growing noise. Much less is known concerning the cases of nonlinear equations with nonlinear noises. Indeed, the interplay between regularization provided by noise and the nonlinearities of the governing equation is more complicated. For example, singularities can be prevented in some cases (cf. [25]: coalescence of vortices disappears in stochastic 2-D Euler equations). On the other hand, it is known that noise does not prevent shock formation in the Burgers equation, see [23].

Therefore the second goal of this work is to study the case of strong nonlinear noise and consider its effect. As we will see in (2.5) below, for the solution to (1.8), its $H^{s}$-norm blows up if and only if its $W^{1, \infty}$-norm blows up. This suggests choosing a noise coefficient involving the $W^{1, \infty}$-norm of $u$. Therefore in this work we consider the case that $h(t, u) \mathrm{d} \mathcal{W}=a\left(1+\|u\|_{W^{1, \infty}}\right)^{\theta} u \mathrm{~d} W$, where $\theta>0, a \in \mathbb{R}$ and $W$ is a standard 1-D Brownian motion. We will try to determine the range of $\theta$ and $a$ such that the solution to the following problem 
exists globally in time:

$$
\left\{\begin{array}{l}
\mathrm{d} u+\left[(u-\gamma) u_{x}+F(u)\right] \mathrm{d} t=a\left(1+\|u\|_{W^{1, \infty}}\right)^{\theta} u \mathrm{~d} W, \quad x \in \mathbb{T}, t>0, \\
u(\omega, 0, x)=u_{0}(\omega, x), \quad x \in \mathbb{T} .
\end{array}\right.
$$

As is shown in Theorem 2.2 below, if the noise is strong enough (either $\theta>1 / 2$, $a \neq 0$ or $\theta=1 / 2, a^{2} \gg 1$ ), then the global existence holds true for (1.10) almost surely. This result justifies the idea that large nonlinear noise can actually prevent blow-up.

On the other hand, as put forward by e.g. Whitham in [51], the wave breaking phenomenon is one of the most intriguing long-standing problems of water wave theory. For the deterministic $\mathrm{CH}$ type equations, the wave breaking phenomenon has been extensively studied, see $[12,13,43]$ for example. Particularly, for equations with dissipation term $\lambda\left(u-u_{x x}\right)$, we refer to [53] for the phenomenon of wave breaking. When random noise is involved, as far as we know, we can only refer to $[17,49]$ for wave breaking. In [17] the authors proved that temporal stochasticity (in the sense of Stratonovich) in the diffeomorphic flow map for the stochastic $\mathrm{CH}$ equation does not prevent the wave breaking process. In [49], wave breaking in the stochastic $\mathrm{CH}$ equation with multiplicative Itô noise is considered.

Thus, the third goal of this paper is to consider noise effects associated with the phenomenon of wave breaking. Due to Theorem 2.2, we see that if wave breaking occurs, the noise term does not grow fast. Hence we consider $\theta=0$ in (1.10) but introduce a non-autonomous pre-factor depending on time $t$. Precisely, we consider the DGH equation with linear multiplicative noise given by

$$
\left\{\begin{array}{l}
\mathrm{d} u+\left[(u-\gamma) \partial_{x} u+F(u)\right] \mathrm{d} t=b(t) u \mathrm{~d} W, \quad x \in \mathbb{T}, t>0, \\
u(\omega, 0, x)=u_{0}(\omega, x), \quad x \in \mathbb{T} .
\end{array}\right.
$$

This case can be formally reformulated as the following stochastic evolution equation when $s>3$

$$
u_{t}-u_{x x t}+c_{0} u_{x}+3 u u_{x}+\gamma u_{x x x}=2 u_{x} u_{x x}+u u_{x x x}+b(t)\left(u-u_{x x}\right) \dot{W} .
$$

When $c_{0}+\gamma=0$, we give two conditions on the initial data that guarantee the global existence of the solutions. Besides, we also estimate the probability that the solution breaks and describe its breaking rate. See Theorems 2.3-2.7 for the statements.

The precise statements of all the results above can be found in Sect. 2 jointly with the necessary assumptions on the noise coefficient.

\section{Definitions, assumptions and main results}

We begin by introducing some notations. $L^{2}(\mathbb{T})$ is the usual space of squareintegrable functions on $\mathbb{T}$. For $s \in \mathbb{R}, D^{s}=\left(1-\partial_{x x}^{2}\right)^{s / 2}$ is defined by $\widehat{D^{s} f}(k)=$ 
$\left(1+k^{2}\right)^{s / 2} \widehat{f}(k)$, where $\widehat{g}$ is the Fourier transform of $g$. The Sobolev space $H^{s}(\mathbb{T})$ is defined as

$$
H^{s}(\mathbb{T}) \triangleq\left\{f \in L^{2}(\mathbb{T}):\|f\|_{H^{s}(\mathbb{T})}^{2}=\sum_{k \in \mathbb{Z}}\left(1+k^{2}\right)^{s}|\widehat{f}(k)|^{2}<\infty\right\},
$$

and the inner product $(f, g)_{H^{s}}$ is $(f, g)_{H^{s}}:=\sum_{k \in \mathbb{Z}}\left(1+k^{2}\right)^{s} \widehat{f}(k) \cdot \overline{\widehat{g}}(k)=$ $\left(D^{s} f, D^{s} g\right)_{L^{2}}$. When the function space refers to $\mathbb{T}$, we will drop $\mathbb{T}$ if there is no ambiguity. We will use $\lesssim$ to denote estimates that hold up to some universal deterministic constant which may change from line to line but whose meaning is clear from the context. For linear operators $A$ and $B$, we denote $[A, B]=A B-B A$.

We briefly recall some aspects of the theory of infinite dimensional stochastic analysis which we will use below. We refer the readers to $[18,26,33]$ for an extended treatment of this subject.

We call $\mathcal{S}=\left(\Omega, \mathcal{F}, \mathbb{P},\left\{\mathcal{F}_{t}\right\}_{t \geq 0}, \mathcal{W}\right)$ a stochastic basis, where $\left\{\mathcal{F}_{t}\right\}_{t \geq 0}$ is a right-continuous filtration on $(\Omega, \mathcal{F})$ such that $\left\{\mathcal{F}_{0}\right\}$ contains all the $\mathbb{P}$ negligible subsets and $\mathcal{W}(t)=\mathcal{W}(\omega, t)(\omega \in \Omega)$ is a cylindrical Wiener process adapted to $\left\{\mathcal{F}_{t}\right\}_{t \geq 0}$. More precisely, we consider a separable Hilbert space $U$ as well as a larger Hilbert space $U_{0}$ such that the embedding $U \hookrightarrow U_{0}$ is Hilbert-Schmidt. Therefore we define

$$
\mathcal{W}=\sum_{k=1}^{\infty} W_{k} e_{k} \in C\left([0, \infty) ; U_{0}\right) \mathbb{P}-\text { a.s. }
$$

where $\left\{W_{k}\right\}_{k \geq 1}$ is a sequence of mutually independent 1-D Brownian motions and $\left\{e_{k}\right\}_{k \in \mathbb{N}}$ is a complete orthonormal basis of $U$.

For a predictable stochastic process $G$ taking values in the space of Hilbert-Schmidt operators from $U$ to $H^{s}$, denoted by $\mathcal{L}_{2}\left(U ; H^{s}\right)$, the Itô stochastic integral

$$
\int_{0}^{\tau} G \mathrm{~d} \mathcal{W}=\sum_{k=1}^{\infty} \int_{0}^{\tau} G e_{k} \mathrm{~d} W_{k}
$$

is well defined (see $[18,44]$ for example). Remember that

$$
G \in \mathcal{L}_{2}\left(U ; H^{s}\right) \Longleftrightarrow\|G\|_{\mathcal{L}_{2}\left(U ; H^{s}\right)}^{2}=\sum_{k=1}^{\infty}\left\|G e_{k}\right\|_{H^{s}}^{2}<\infty .
$$

The stochastic integral $\int_{0}^{t} G \mathrm{~d} \mathcal{W}$ is an $H^{s}$-valued square-integrable martingale. In our case we have the Burkholder-Davis-Gundy inequality

$$
\mathbb{E}\left(\sup _{t \in[0, T]}\left\|\int_{0}^{t} G \mathrm{~d} \mathcal{W}\right\|_{H^{s}}^{p}\right) \leq C(p, s) \mathbb{E}\left(\int_{0}^{T}\|G\|_{\mathcal{L}_{2}\left(U ; H^{s}\right)}^{2} \mathrm{~d} t\right)^{\frac{p}{2}}, \quad p \geq 1 .
$$

\subsection{Definitions of the solutions}

We now precise the notion of pathwise solutions to (1.8). 
Definition 2.1. [Pathwise solutions] Let $\mathcal{S}=\left(\Omega, \mathcal{F}, \mathbb{P},\left\{\mathcal{F}_{t}\right\}_{t \geq 0}, \mathcal{W}\right)$ be a fixed stochastic basis. Let $s>3 / 2$ and $u_{0}$ be an $H^{s}$-valued $\mathcal{F}_{0}$ measurable random variable.

(1) A local pathwise solution to (1.8) is a pair $(u, \tau)$, where $\tau$ is a stopping time satisfying $\mathbb{P}\{\tau>0\}=1$ and $u: \Omega \times[0, \infty) \rightarrow H^{s}$ is an $\mathcal{F}_{t}$ predictable $H^{s}$-valued process satisfying

$$
u(\cdot \wedge \tau) \in C\left([0, \infty) ; H^{s}\right) \mathbb{P}-a . s .,
$$

and for all $t>0$,

$$
u(t \wedge \tau)-u(0)+\int_{0}^{t \wedge \tau}\left[(u-\gamma) \partial_{x} u+F(u)\right] \mathrm{d} t^{\prime}=\int_{0}^{t \wedge \tau} h\left(t^{\prime}, u\right) \mathrm{d} \mathcal{W} \mathbb{P}-\text { a.s. }
$$

(2) Local pathwise solutions are said to be pathwise unique, if given any two pairs of local pathwise solutions $\left(u_{1}, \tau_{1}\right)$ and $\left(u_{2}, \tau_{2}\right)$ with $\mathbb{P}\left\{u_{1}(0)=\right.$ $\left.u_{2}(0)\right\}=1$, we have

$$
\mathbb{P}\left\{u_{1}(t, x)=u_{2}(t, x), \quad \forall(t, x) \in\left[0, \tau_{1} \wedge \tau_{2}\right] \times \mathbb{T}\right\}=1 .
$$

(3) Additionally, $\left(u, \tau^{*}\right)$ is called a maximal solution to (1.8) if $\tau^{*}>0$ almost surely and if there is an increasing sequence $\tau_{n} \rightarrow \tau^{*}$ such that for any $n \in \mathbb{N},\left(u, \tau_{n}\right)$ is a pathwise solution to (1.8) and on the set $\left\{\tau^{*}<\infty\right\}$,

$$
\sup _{t \in\left[0, \tau_{n}\right]}\|u\|_{H^{s}} \geq n \text {. }
$$

(4) If $\tau^{*}=\infty$ almost surely, then we say that the pathwise solution exists globally.

\subsection{Assumptions}

Next, we prescribe some conditions on the noise coefficient $h$ in (1.8) and on $b$ in (1.12).

Assumption 2.1. Let $s>1 / 2$. We assume that $h:[0, \infty) \times H^{s} \ni(t, u) \mapsto$ $h(t, u) \in \mathcal{L}_{2}\left(U ; H^{s}\right)$ is continuous in $(t, u)$. Moreover, we assume the following:

- There is a non-decreasing locally bounded function $f(\cdot):[0, \infty) \rightarrow[0, \infty)$ such that for any $t>0$,

$$
\|h(t, u)\|_{\mathcal{L}_{2}\left(U ; H^{s}\right)} \leq f\left(\|u\|_{W^{1, \infty}}\right)\|u\|_{H^{s}} .
$$

Particularly, in the additive noise case, we assume $h:[0, \infty) \times \mathbb{T} \ni$ $(t, x) \mapsto h(t, x) \in \mathcal{L}_{2}\left(U ; H^{s}\right)$ is continuous meaning that (2.2) reduces to $\|h(t, x)\|_{\mathcal{L}_{2}\left(U ; H^{s}\right)} \leq C$ for some $C>0$.

- There is a non-decreasing locally bounded function $q(\cdot):[0, \infty) \rightarrow[0, \infty)$, such that for any $t>0$,

$$
\sup _{\|u\|_{H^{s}},\|v\|_{H^{s}} \leq N}\left\{\mathbf{1}_{\{u \neq v\}} \frac{\|h(t, u)-h(t, v)\|_{\mathcal{L}_{2}\left(U ; H^{s}\right)}}{\|u-v\|_{H^{s}}}\right\} \leq q(N), \quad N \geq 1 .
$$

After the regularization effect of strong noise is established in Theorem 2.2 , to analyze the effect of noise on the regularity of pathwise solutions, we restrict ourselves to the linear-noise case (1.12) imposing the following bounds on the coefficient $b$. 
Assumption 2.2. When considering (1.12) with non-autonomous linear noise $b(t) u \mathrm{~d} W$, we assume that there are constants $b_{*}, b^{*}>0$ such that $0<b_{*} \leq$ $b^{2}(t) \leq b^{*}$ for all $t$.

Remark 2.1. Let us give some brief explanations for the assumptions.

- The function $h:[0, \infty) \times H^{s} \ni(t, u) \mapsto h(t, u) \in \mathcal{L}_{2}\left(U ; H^{s}\right)$ is required to be continuous in $(t, u)$. This will be essential to pass to the limit when establishing the existence of a martingale solution as an intermediate step, cf. $[3,48,49]$.

- The uniform-in-time assumption (2.2) bounds the growth of the $\mathcal{L}_{2}(U$; $H^{s}$ )-norm of the noise coefficient in terms of a product of a nonlinear function of the $W^{1, \infty}$-norm and the $H^{s}$-norm. This allows us to control the $W^{1, \infty}$-norm by some cut-off later.

- Formula (2.3) ensures local Lipschitz continuity in $H^{s}$, which will be used to obtain (local) existence and uniqueness.

- Let us outline that we will use a Girsanov-type transformation to study (1.11) (see Remark 2.6 and Sect. 6). The assumption $b^{2}(t) \leq b^{*}$ is used to guarantee that such transformation is well-defined and the condition $b(t) \neq 0, t \geq 0$ is needed to establish certain estimates for the Girsanovtype process e $\mathrm{e}_{0}^{t} b\left(t^{\prime}\right) \mathrm{d} W_{t^{\prime}}-\int_{0}^{t} \frac{b^{2}\left(t^{\prime}\right)}{2} \mathrm{~d} t^{\prime}$ (see Lemma 3.7). In Theorem 2.3, the condition $0<b_{*} \leq b^{2}(0)$ is used to bound the initial data, cf. (2.7).

\subsection{Main results and remarks}

Now we present our results. For the general case (1.8), we have the following local existence result which moreover relates the possible blow-up in the $H^{s}$ norm to simultaneous blow up in the $W^{1, \infty}$-norm.

Theorem 2.1. (Maximal solutions) Let $s>3 / 2, c_{0}, \gamma \in \mathbb{R}$ and let $h(t, u)$ satisfy Assumption 2.1. For a given stochastic basis $\mathcal{S}=\left(\Omega, \mathcal{F}, \mathbb{P},\left\{\mathcal{F}_{t}\right\}_{t \geq 0}, \mathcal{W}\right)$ and an $H^{s}$-valued $\mathcal{F}_{0}$ measurable random variable $u_{0}$ satisfying $\mathbb{E}\left\|u_{0}\right\|_{H^{s}}^{2}<\infty$, the initial value problem (1.8) admits a local unique pathwise solution $(u, \tau)$ in the sense of Definition 2.1 with

$$
u(\cdot \wedge \tau) \in L^{2}\left(\Omega ; C\left([0, \infty) ; H^{s}\right)\right) .
$$

Besides, $(u, \tau)$ can be extended to a unique maximal solution $\left(u, \tau^{*}\right)$ in the sense of Definition 2.1 and the following blow-up criterion holds true:

$$
1_{\left\{\lim \sup _{t \rightarrow \tau^{*}}\|u(t)\|_{H^{s}}=\infty\right\}}=1_{\left\{\lim \sup _{t \rightarrow \tau^{*}}\|u(t)\|_{W^{1, \infty}}=\infty\right\}} \mathbb{P}-\text { a.s. }
$$

Remark 2.2. For the proof of Theorem 2.1 one can follow the ideas in e.g. $[2-4,16,19,29,48]$ by constructing a sequence of approximations for a problem with cut-off for the $W^{1, \infty}$-norm. Such a cut-off implies at-most linear growth of $u$ and guarantees the global existence of an approximate solution. Otherwise we have to find a positive lower bound for the lifespan of the approximate solutions, which is a priori not clear. Besides, with the cut-off, one can close the a priori $L^{2}\left(\Omega ; H^{s}\right)$ estimate by splitting $\mathbb{E}\left(\|u\|_{H^{s}}^{2}\|u\|_{W^{1, \infty}}\right)$.

Turning to noise-driven regularization effects, the blow-up criterion (2.5) suggests relating the noise coefficient to the $W^{1, \infty}$-norm of $u$. Therefore we 
consider (1.10) with scalable noise impact, i.e., we assume $h(t, u)=a(1+$ $\|u\|)_{W^{1, \infty}}^{\theta} u$ for some $\theta>0$ and $a \in \mathbb{R}$. When $a$ and $\theta$ satisfy certain strengthconditions, the noise term counteracts the formation of singularities and we have

Theorem 2.2. (Global existence for strong nonlinear noise) Let $\mathcal{S}=(\Omega, \mathcal{F}, \mathbb{P}$, $\left.\left\{\mathcal{F}_{t}\right\}_{t \geq 0}, W\right)$ be a fixed stochastic basis. Let $s>\frac{5}{2}, c_{0}, \gamma \in \mathbb{R}$ and $u_{0} \in H^{s}$ be an $H^{s}$-valued $\mathcal{F}_{0}$-measurable random variable with $\mathbb{E}\left\|u_{0}\right\|_{H^{s}}^{2}<\infty$. Assume that $\theta$ and a satisfy

$$
\text { either } a \in \mathbb{R} \backslash\{0\}, \theta>\frac{1}{2} \quad \text { or } \quad a^{2}>2 Q, \theta=\frac{1}{2},
$$

where $Q=Q\left(s, c_{0}, \gamma\right)$ is a constant that will be specified in Lemma 3.5. Then the corresponding maximal solution $\left(u, \tau^{*}\right)$ to (1.10) satisfies

$$
\mathbb{P}\left\{\tau^{*}=\infty\right\}=1 \text {. }
$$

Theorem 2.2 implies that blow-up of pathwise solutions might only be observed if the noise is weak. To detect such noise, we analyze the simpler ansatz $h(t, u)=b(t) u$ as in (1.11). Even in this linear noise case the situation is quite subtle allowing for global existence as well as blow-up of solutions. For global existence, we can identify two cases.

Theorem 2.3. (Global existence for weak noise I) Let $s>3 / 2$. Assume $c_{0}+\gamma=$ 0 . Let $b(t)$ satisfy Assumption 2.2 and $\mathcal{S}=\left(\Omega, \mathcal{F}, \mathbb{P},\left\{\mathcal{F}_{t}\right\}_{t \geq 0}, W\right)$ be a fixed stochastic basis. Assume $u_{0}$ is an $H^{s}$-valued $\mathcal{F}_{0}$ measurable random variable satisfying $\mathbb{E}\left\|u_{0}\right\|_{H^{s}}^{2}<\infty$. Let $K=K(s)>0$ be a constant such that the embedding $\|\cdot\|_{W^{1, \infty}}<K\|\cdot\|_{H^{s}}$ holds. Then there is a $C=C(s)>1$ such that for any $R>1$ and $\lambda_{1}>2$, if

$$
\left\|u_{0}\right\|_{H^{s}}<\frac{b_{*}}{C K \lambda_{1} R} \quad \mathbb{P}-\text { a.s. },
$$

then (1.11) has a maximal solution $\left(u, \tau^{*}\right)$ satisfying for any $\lambda_{2}>\frac{2 \lambda_{1}}{\lambda_{1}-2}$ the estimate

$\mathbb{P}\left\{\|u(t)\|_{H^{s}}<\frac{b_{*}}{C K \lambda_{1}} \mathrm{e}^{-\frac{\left(\left(\lambda_{1}-2\right) \lambda_{2}-2 \lambda_{1}\right)}{2 \lambda_{1} \lambda 2} \int_{0}^{t} b^{2}\left(t^{\prime}\right) \mathrm{d} t^{\prime}}\right.$ for all $\left.t>0\right\} \geq 1-\left(\frac{1}{R}\right)^{2 / \lambda_{2}}$.

Theorem 2.4. (Global existence for weak noise II) Let $c_{0}+\gamma=0$ and $s>3$. Let $b(t)$ satisfy Assumption 2.2 and $\mathcal{S}=\left(\Omega, \mathcal{F}, \mathbb{P},\left\{\mathcal{F}_{t}\right\}_{t \geq 0}, W\right)$ be a fixed stochastic basis. Assume $u_{0}$ is an $H^{s}$-valued $\mathcal{F}_{0}$ measurable random variable satisfying $\mathbb{E}\left\|u_{0}\right\|_{H^{s}}^{2}<\infty$. If $u_{0}$ satisfies

$$
\begin{aligned}
& \mathbb{P}\left\{\left(1-\partial_{x x}^{2}\right) u_{0}(x)>0, \quad \forall x \in \mathbb{T}\right\}=p, \\
& \mathbb{P}\left\{\left(1-\partial_{x x}^{2}\right) u_{0}(x)<0, \quad \forall x \in \mathbb{T}\right\}=q,
\end{aligned}
$$

for some $p, q \in[0,1]$, then the corresponding maximal solution $\left(u, \tau^{*}\right)$ to $(1.11)$ satisfies

$$
\mathbb{P}\left\{\tau^{*}=\infty\right\} \geq p+q
$$


Remark 2.3. Theorem 2.3 provides a global existence result for initial data with bounded $H^{s}$-norm depending on the strength of the noise. This result can not be observed in the deterministic case because $0<b_{*} \leq b^{2}(t)$ is required (see Assumption 2.2). On the other hand, since the proof of Theorem 2.4 relies on the analysis of a PDE with random coefficient (see (6.2) below), the deterministic case can be included by formally letting the random coefficient be 1 . Therefore, in this sense, Theorem 2.4 covers the corresponding deterministic result, cf. $[13,41]$. Indeed, by letting $\beta \equiv 1$ in $(6.2)$ and taking $(p, q)=(1,0)$ or $(p, q)=(0,1)$ in Theorem 2.4 , we obtain the global existence for the deterministic DGH equation.

According to (2.5) in Theorem 2.1, a blow-up comes along with an explosion of the $W^{1, \infty}$-norm. For the special noise in (1.11) we can improve the result by showing that a blow-up is related to the first spatial derivative only and corresponds to the wave-breaking phenomenon with exploding negative slope.

Theorem 2.5. (Blow-up scenario) Let $c_{0}+\gamma=0, s>3$ and Assumption 2.2 be satisfied. Let $\mathcal{S}=\left(\Omega, \mathcal{F}, \mathbb{P},\left\{\mathcal{F}_{t}\right\}_{t \geq 0}, W\right)$ be fixed in advance. Let $\left(u, \tau^{*}\right)$ be the unique maximal solution to (1.11) starting from an $\mathcal{F}_{0}$ measurable random variable $u_{0} \in L^{2}\left(\Omega ; H^{s}\right)$. Then the singularities can arise only in the form of wave breaking, i.e.,

$$
\mathbb{P}\left\{\|u(t)\|_{L^{\infty}} \lesssim A\left\|u_{0}\right\|_{H^{1}}<\infty, \quad \forall t>0\right\}=1,
$$

where $A=A(\omega)=\sup _{t>0} \mathrm{e}^{\int_{0}^{t} b\left(t^{\prime}\right) \mathrm{d} W_{t^{\prime}}-\int_{0}^{t} \frac{b^{2}\left(t^{\prime}\right)}{2} \mathrm{~d} t^{\prime}}<\infty \mathbb{P}-$ a.s., and

$$
1_{\left\{\lim \sup _{t \rightarrow \tau^{*}}\|u(t)\|_{H^{s}}=\infty\right\}}=1_{\left\{\lim _{\inf } \operatorname{li}_{t \rightarrow}\left[\min _{x \in \mathbb{T}} u_{x}(t, x)\right]=-\infty\right\}} \mathbb{P}-\text { a.s. }
$$

Still we have not identified initial data for (1.11) that lead to a blow-up. A precise condition in terms of probability is given in the next theorem. To formulate it, we introduce the number $\lambda>0$ such that for any $f \in H^{3}$, the estimate

$$
\max _{x \in \mathbb{T}} f^{2}(x) \leq \lambda\|f\|_{H^{1}}^{2}
$$

holds.

Theorem 2.6. (Wave breaking and its probability) Let $\mathcal{S}=\left(\Omega, \mathcal{F}, \mathbb{P},\left\{\mathcal{F}_{t}\right\}_{t \geq 0}\right.$, $W)$ be a fixed stochastic basis, $c_{0}+\gamma=0$ and $s>3$. Let Assumption 2.2 be verified and let $u_{0} \in L^{2}\left(\Omega ; H^{s}\right)$ be $\mathcal{F}_{0}$ measurable. If for some $c \in(0,1)$,

$$
\min _{x \in \mathbb{T}} \partial_{x} u_{0}(x)<-\frac{1}{2} \sqrt{\frac{\left(b^{*}\right)^{2}}{c^{2}}+4 \lambda\left\|u_{0}\right\|_{H^{1}}^{2}}-\frac{b^{*}}{2 c} \mathbb{P}-\text { a.s. },
$$

where $b^{*}$ is given in Assumption 2.2 and $\lambda$ is given in (2.11), then the maximal solution $\left(u, \tau^{*}\right)$ to (1.11) (or (1.12), equivalently) satisfies

$$
\mathbb{P}\left\{\tau^{*}<\infty\right\} \geq \mathbb{P}\left\{\mathrm{e}^{\int_{0}^{t} b\left(t^{\prime}\right) \mathrm{d} W_{t^{\prime}}}>c, \quad \forall t>0\right\}>0 .
$$

By Theorem 2.5, we have $\mathbb{P}\{u$ breaks in finite time $\} \geq \mathbb{P}\left\{\mathrm{e}^{\int_{0}^{t} b\left(t^{\prime}\right) \mathrm{d} W_{t^{\prime}}}>c\right.$, $\forall t>0\}>0$. 
Remark 2.4. Whereas Theorem 2.3 provides a global existence result, Theorem 2.6 detects the formation of singularities in finite time under certain conditions on the initial data. We stress that these two conditions are mutually exclusive. In Theorem 2.3 we suppose $\left\|u_{0}\right\|_{H^{s}} \leq \frac{b_{*}}{C K \lambda_{1} R}$ with $C>1, \lambda_{1}>2$ and $R>1$ almost surely. Then $u$ satisfies (2.8). In Theorem 2.6 we suppose for some $c \in(0,1)$ that $\min _{x \in \mathbb{T}} \partial_{x} u_{0}(x)<-\frac{1}{2} \sqrt{\frac{\left(b^{*}\right)^{2}}{c^{2}}+4 \lambda\left\|u_{0}\right\|_{H^{1}}^{2}}-\frac{b^{*}}{2 c}<-\frac{b^{*}}{2 c}$ almost surely holds. But this means $\left\|u_{0}\right\|_{H^{s}}>\frac{1}{K}\left\|u_{0}\right\|_{W^{1, \infty}} \geq \frac{1}{K}\left|\min _{x \in \mathbb{T}} \partial_{x} u_{0}(x)\right|>$ $\frac{b^{*}}{2 c K}>\frac{b_{*}}{C K \lambda_{1} R}$.

We conclude this section with a result refining Theorem 2.5. It is possible to quantify the blow-up rate.

Theorem 2.7. (Wave breaking rate) Let the conditions in Theorem 2.5 hold true. Then

$$
\lim _{t \rightarrow \tau^{*}}\left(\min _{x \in \mathbb{T}}\left[u_{x}(t, x)\right] \int_{t}^{\tau^{*}} \beta\left(t^{\prime}\right) \mathrm{d} t^{\prime}\right)=-2 \beta\left(\tau^{*}\right) \quad \text { a.e. on } \quad\left\{\tau^{*}<\infty\right\}
$$

where

$$
\beta(\omega, t)=\mathrm{e}^{\int_{0}^{t} b\left(t^{\prime}\right) \mathrm{d} W_{t^{\prime}}-\int_{0}^{t} \frac{b^{2}\left(t^{\prime}\right)}{2} \mathrm{~d} t^{\prime}} .
$$

Remark 2.5. As a corollary of Theorems 2.5 and 2.7, we have that as long as singularities occurs, they can arise only in the form of wave breaking and the breaking rate is given by (2.12). This result is optimal in the sense that it is consistent with the result for the corresponding deterministic case. Indeed, for the deterministic DGH equation (cf. [41, Theorem 4.2]), the blow-up rate is

$$
\lim _{t \rightarrow \tau^{*}}\left(\min _{x \in \mathbb{T}}\left[u_{x}(t, x)\right]\left(\tau^{*}-t\right)\right)=-2 .
$$

Formally, since the deterministic DGH equation can be viewed as (6.2) with $\beta \equiv 1$, we see that the blow-up estimate (2.12) coincides with the above deterministic result when $\beta \equiv 1$.

Remark 2.6. Let us make a comment on the idea for the subsequent analysis of (1.11), which is motivated by $[29,46,48]$. By introducing the Girsanov-type transformation

$$
v=\frac{1}{\beta(\omega, t)} u, \quad \beta(\omega, t)=\mathrm{e}^{\int_{0}^{t} b\left(t^{\prime}\right) \mathrm{d} W_{t^{\prime}}-\int_{0}^{t} \frac{b^{2}\left(t^{\prime}\right)}{2} \mathrm{~d} t^{\prime}},
$$

we obtain an equation for $v$ (see Sect. 6 for the detailed calculation), namely $v_{t}+\beta v v_{x}-\gamma v_{x}+\beta\left(1-\partial_{x x}^{2}\right)^{-1} \partial_{x}\left(v^{2}+\frac{1}{2} v_{x}^{2}\right)+\left(c_{0}+\gamma\right)\left(1-\partial_{x x}^{2}\right)^{-1} \partial_{x} v=0$

Although the above equation for $v$ does not depend on a stochastic integral on $v$ itself, to extend the deterministic results to the stochastic setting, we need to overcome a few technical difficulties since the system is not only random but also non-autonomous (see e.g., (6.6), (6.8) and (6.10)). With the help of certain estimates and asymptotic limits of Girsanov-type processes (see Lemma 3.7), we are able to apply the energy estimate pathwisely (for a.e. $\omega \in \Omega$ ) to study the global existence and possible blow-up of solutions. 
We outline the rest of the paper. In the next section, we briefly recall some relevant preliminaries. In Sect. 4, we prove Theorem 2.1. For the large noise case, we prove Theorem 2.2 in Sect. 5. For the non-autonomous linear multiplicative noise case, we consider the global existence, decay, wave breaking and the blow-up rate of the pathwise solutions and prove Theorems 2.3, 2.4, 2.6 and 2.7 in Sect. 6.

\section{Preliminary results}

We summarize some auxiliary results, which will be used to prove our main results from Sect. 2. Define the regularizing operator $T_{\varepsilon}$ on $\mathbb{T}$ as

$$
T_{\varepsilon} f(x):=\left(1-\varepsilon^{2} \Delta\right)^{-1} f(x)=\sum_{k \in \mathbb{Z}}\left(1+\varepsilon^{2}|k|^{2}\right)^{-1} \widehat{f}(k) \mathrm{e}^{\mathrm{i} x k}, \quad \varepsilon \in(0,1) .
$$

Since $T_{\varepsilon}$ can be characterized by its Fourier multipliers, it is easy to see

$$
\begin{aligned}
{\left[D^{s}, T_{\varepsilon}\right] } & =0, \\
\left(T_{\varepsilon} f, g\right)_{L^{2}} & =\left(f, T_{\varepsilon} g\right)_{L^{2}}, \\
\left\|T_{\varepsilon} u\right\|_{H^{s}} & \leq\|u\|_{H^{s}} .
\end{aligned}
$$

Furthermore, we have

Lemma 3.1. ([49]) Let $f, g: \mathbb{T} \rightarrow \mathbb{R}$ such that $g \in W^{1, \infty}$ and $f \in L^{2}$. Then for some $C>0$,

$$
\left\|\left[T_{\varepsilon}, g\right] f_{x}\right\|_{L^{2}} \leq C\|g\|_{W^{1, \infty}}\|f\|_{L^{2}} .
$$

The following estimates are classical for Sobolev spaces.

Lemma 3.2. ([34]) Let $s>1$. There is a $C_{s}>0$ such that for all $f \in H^{s} \cap$ $W^{1, \infty}, g \in H^{s-1} \cap L^{\infty}$ we have

$$
\left\|\left[D^{s}, f\right] g\right\|_{L^{2}} \leq C_{s}\left(\left\|D^{s} f\right\|_{L^{2}}\|g\|_{L^{\infty}}+\left\|\partial_{x} f\right\|_{L^{\infty}}\left\|D^{s-1} g\right\|_{L^{2}}\right) .
$$

Lemma 3.3. ([34]) Let $s>0$, then there is a $C_{s}>0$ such that we have for all $f, g \in H^{s} \cap L^{\infty}$ the estimate

$$
\|f g\|_{H^{s}} \leq C_{s}\left(\|f\|_{H^{s}}\|g\|_{L^{\infty}}+\|f\|_{L^{\infty}}\|g\|_{H^{s}}\right) .
$$

Specifically, for our problem (1.8), we have introduced the nonlocal term $F(\cdot)$ in (1.9). Using the Moser estimate from Lemma 3.3, we can obtain the next statement on $F(\cdot)$ (see [50]).

Lemma 3.4. For $F(\cdot)$ defined in (1.9) and for any $v, v_{1}, v_{2} \in H^{s}$ with $s>1 / 2$, we have

$$
\begin{aligned}
&\|F(v)\|_{H^{s}} \lesssim\left(\|v\|_{L^{\infty}}+\left\|\partial_{x} v\right\|_{L^{\infty}}+\left(c_{0}+\gamma\right)\right)\|v\|_{H^{s}}, \quad s>3 / 2 \\
&\left\|F\left(v_{1}\right)-F\left(v_{2}\right)\right\|_{H^{s}} \lesssim\left(\left\|v_{1}\right\|_{H^{s}}+\left\|v_{2}\right\|_{H^{s}}+\left(c_{0}+\gamma\right)\right)\left\|v_{1}-v_{2}\right\|_{H^{s}}, \quad s>3 / 2 \\
&\left\|F\left(v_{1}\right)-F\left(v_{2}\right)\right\|_{H^{s}} \lesssim\left(\left\|v_{1}\right\|_{H^{s+1}}+\left\|v_{2}\right\|_{H^{s+1}}+\left(c_{0}+\gamma\right)\right)\left\|v_{1}-v_{2}\right\|_{H^{s}}, \\
& 3 / 2 \geq s>1 / 2 .
\end{aligned}
$$


The following estimate will be used in the proof of the blow-up criterion (2.5) and of Theorem 2.2.

Lemma 3.5. Let $s>3 / 2, c_{0}, \gamma \in \mathbb{R}$. Let $F(\cdot)$ and $T_{\varepsilon}$ be given in (1.9) and (3.1), respectively. There is a constant $Q=Q\left(s, c_{0}, \gamma\right)>0$ such that for all $\varepsilon>0$,

$$
\left|\left(T_{\varepsilon}\left[(u-\gamma) u_{x}\right], T_{\varepsilon} u\right)_{H^{s}}\right|+\left|\left(T_{\varepsilon} F(u), T_{\varepsilon} u\right)_{H^{s}}\right| \leq Q\left(1+\|u\|_{W^{1, \infty}}\right)\|u\|_{H^{s}}^{2} .
$$

Proof. We first notice that

$$
\begin{aligned}
& \left(T_{\varepsilon}\left[(u-\gamma) u_{x}\right], T_{\varepsilon} u\right)_{H^{s}}=\int_{\mathbb{T}} D^{s} T_{\varepsilon}\left[(u-\gamma) u_{x}\right] \cdot D^{s} T_{\varepsilon} u \mathrm{~d} x \\
& \quad=\int_{\mathbb{T}} D^{s} T_{\varepsilon}\left[u u_{x}\right] \cdot D^{s} T_{\varepsilon} u \mathrm{~d} x .
\end{aligned}
$$

Due to (3.2) and (3.3), we commute the operator to derive

$$
\begin{aligned}
& \left(D^{s} T_{\varepsilon}\left[u u_{x}\right], D^{s} T_{\varepsilon} u\right)_{L^{2}} \\
& \quad=\left(\left[D^{s}, u\right] u_{x}, D^{s} T_{\varepsilon}^{2} u\right)_{L^{2}}+\left(\left[T_{\varepsilon}, u\right] D^{s} u_{x}, D^{s} T_{\varepsilon} u\right)_{L^{2}}+\left(u D^{s} T_{\varepsilon} u_{x}, D^{s} T_{\varepsilon} u\right)_{L^{2}} .
\end{aligned}
$$

Then it follows from Lemmas 3.1 and 3.2, integration by parts, (3.4) and $H^{s} \hookrightarrow W^{1, \infty}$ that

$$
\left|\left(T_{\varepsilon}\left[(u-\gamma) u_{x}\right], T_{\varepsilon} u\right)_{H^{s}}\right| \lesssim\|u\|_{W^{1, \infty}}\|u\|_{H^{s}}^{2} .
$$

Using Lemma 3.4 and (3.4) directly, we have

$$
\left|\left(T_{\varepsilon} F(u), T_{\varepsilon} u\right)_{H^{s}}\right| \lesssim\left(\|u\|_{W^{1, \infty}}+\left(c_{0}+\gamma\right)\right)\|u\|_{H^{s}}^{2} .
$$

Combining the above two inequalities gives rise to the desired estimate of the lemma.

The following lemma has been established for the real-line case in [12] and [10], respectively. They hold likewise for $x \in \mathbb{T}$, using the periodicity on $\mathbb{T}$.

Lemma 3.6. Let $T>0$ and $u \in C^{1}\left([0, T) ; H^{2}(\mathbb{T})\right)$. Then given any $t \in[0, T)$, there is at least one point $z(t)$ with

$$
M(t):=\min _{x \in \mathbb{T}}\left[u_{x}(t, x)\right]=u_{x}(t, z(t)) .
$$

Moreover, the function $M=M(t)$ is almost everywhere differentiable on $(0, T)$ with

$$
\frac{\mathrm{d}}{\mathrm{d} t} M(t)=u_{t x}(t, z(t)) \text { a.e. on }(0, T) .
$$

We conclude this preparatory section with some results from [47], which are needed to establish the theorems on global existence.

Lemma 3.7. Let Assumption 2.2 hold true and assume that $a(t) \in C([0, \infty))$ is a bounded function. For

$$
X=\mathrm{e}^{\int_{0}^{t} b\left(t^{\prime}\right) \mathrm{d} W_{t^{\prime}}+\int_{0}^{t} a\left(t^{\prime}\right)-\frac{b^{2}\left(t^{\prime}\right)}{2} \mathrm{~d} t^{\prime}}
$$

the following properties hold true. 
(i) Let $\phi(t):=\int_{0}^{t} b^{2}\left(t^{\prime}\right) \mathrm{d} t^{\prime}$ with inverse $\phi^{-1}(t)$. If

$$
\limsup _{t \rightarrow \infty} \frac{1}{\sqrt{2 t \log \log t}}\left(\int_{0}^{\phi^{-1}(t)} a\left(t^{\prime}\right) \mathrm{d} t^{\prime}-\frac{t}{2}\right)<-1,
$$

then

$$
\lim _{t \rightarrow \infty} X(t)=0 \quad \mathbb{P}-\text { a.s. }
$$

If

$$
\liminf _{t \rightarrow \infty} \frac{1}{\sqrt{2 t \log \log t}}\left(\int_{0}^{\phi^{-1}(t)} a\left(t^{\prime}\right) \mathrm{d} t^{\prime}-\frac{t}{2}\right)>1
$$

then

$$
\lim _{t \rightarrow \infty} X(t)=\infty \mathbb{P}-\text { a.s. }
$$

(ii) Let $a(t)=\lambda b^{2}(t)$ with $\lambda<\frac{1}{2}$ and $\tau_{R}=\inf \{t \geq 0: X(t)>R\}$ with $R>1$, then

$$
\mathbb{P}\left(\tau_{R}=\infty\right) \geq 1-\left(\frac{1}{R}\right)^{1-2 \lambda}
$$

\section{Proof of Theorem 2.1}

We consider the initial value problem (1.8). The proof of existence and uniqueness of pathwise solutions can be carried out by standard procedures used in many works, see $[2,3,28,29,47-49]$ for more details. Therefore we only give a sketch.

(1) Firstly, one constructs a suitable approximation scheme using a cut-off function to control the $W^{1, \infty}$-norm (arising from $(u-\gamma) u_{x},(2.2)$ and Lemma 3.4). With such cut-off, both the drift and diffusion coefficients in the problem become locally Lipschitz continuous and grow linearly in $u$ (cf. [47-49]). Thus the approximation solutions exist globally. Besides, such cut-off enables us to close the a priori $L^{2}\left(\Omega ; H^{s}\right)$ estimate by splitting $\mathbb{E}\left(\|u\|_{H^{s}}^{2}\|u\|_{W^{1, \infty}}\right)$. Therefore by using Lemma 3.2 and (2.2), uniform estimates for the approximation solutions can be established. We refer the readers to $[48,49]$ for some closely related models;

(2) Secondly, by the uniform estimates, one obtains the tightness of the distributions of the approximation solution in $\mathscr{P}\left(C\left([0, T] ; H^{s-1}\right)\right)$, where $\mathscr{P}\left(C\left([0, T] ; H^{s-1}\right)\right)$ is the collection of Borel probability measures on $C\left([0, T] ; H^{s-1}\right)$. We refer to $[45,48,49]$ for example. Applying the probabilistic compactness arguments, i.e., the Prokhorov theorem and the Skorokhod theorem, and using some technical convergence results as in [1-3,19], one verifies the existence of a martingale solution in $H^{s}$ with $s>3$. In this step $s>3$ is an intermediate requirement because the convergence is in $H^{s-1}$ and we need to control the $W^{1, \infty}$-norm by the embedding $H^{s-1} \hookrightarrow W^{1, \infty}$; 
(3) Thirdly, by Lemma 3.4 and (2.3), one can show that pathwise uniqueness holds. Then the Gyöngy-Krylov characterization of the convergence in probability (see [30]) can be applied to show the existence and uniqueness of a pathwise solution in $H^{s}$ with $s>3$, cf. [3, 29,49];

(4) Finally, mollifying initial data, analyzing the convergence and employing the argument as in $[28,29,48,49]$ lead to a local pathwise solution $(u, \tau)$ to $(1.6)$ with $u(\cdot \wedge \tau) \in L^{2}\left(\Omega ; C\left([0, \infty) ; H^{s}\right)\right)$ for $u_{0} \in L^{2}\left(\Omega ; H^{s}\right)$ with $s>3 / 2$.

To finish the proof of Theorem 2.1, we only need to verify the blow-up criterion (2.5). Motivated by [16,47], we first consider, in next lemma, the relationship between the explosion time of $\|u(t)\|_{H^{s}}$ and the explosion time of $\|u(t)\|_{W^{1, \infty}}$ for (1.8). The results of the lemma will not only immediately imply the blow-up criterion (2.5) but also be used in the next sections.

Lemma 4.1. Let $\left(u, \tau^{*}\right)$ be the unique maximal solution to (1.8). Then the

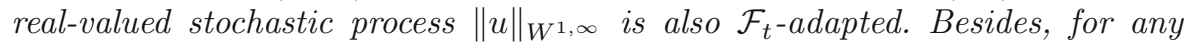
$m, n \in \mathbb{N}$, define

$$
\tau_{1, m}=\inf \left\{t \geq 0:\|u(t)\|_{H^{s}} \geq m\right\}, \quad \tau_{2, n}=\inf \left\{t \geq 0:\|u(t)\|_{W^{1, \infty}} \geq n\right\} .
$$

For $\tau_{1}=\lim _{m \rightarrow \infty} \tau_{1, m}$ and $\tau_{2}=\lim _{n \rightarrow \infty} \tau_{2, n}$, we have then

$$
\tau_{1}=\tau_{2} \mathbb{P}-\text { a.s. }
$$

Proof. To begin with, since $u(\cdot \wedge \tau) \in C\left([0, \infty) ; H^{s}\right)$ almost surely, we see that for any $t \in[0, \tau]$,

$$
[u(t)]^{-1}(Y)=[u(t)]^{-1}\left(H^{s} \cap Y\right), \quad \forall Y \in \mathcal{B}\left(W^{1, \infty}\right) .
$$

Therefore $u(t)$, as a $W^{1, \infty}$-valued process, is also $\mathcal{F}_{t}$-adapted. Moreover, the embedding $H^{s} \hookrightarrow W^{1, \infty}$ for $s>3 / 2$ means that there is a $K=K(s)>0$ such that $\|\cdot\|_{W^{1, \infty}}<K\|\cdot\|_{H^{s}}$. Then for every $m \in \mathbb{N}$,

$$
\sup _{t \in\left[0, \tau_{1, m}\right]}\|u(t)\|_{W^{1, \infty}} \leq K \sup _{t \in\left[0, \tau_{1, m}\right]}\|u(t)\|_{H^{s}} \leq([K]+1) m
$$

where $[K]$ means the integer part of $K$. Consequently, $\tau_{1, m} \leq \tau_{2,([K]+1) m} \leq \tau_{2}$ almost surely, which means that $\tau_{1} \leq \tau_{2} \mathbb{P}-$ a.s. Now we only need to prove the contrary inequality. Let $n, k \in \mathbb{N}$, one has

$$
\begin{aligned}
\left\{\sup _{t \in\left[0, \tau_{2, n} \wedge k\right]}\|u(t)\|_{H^{s}}<\infty\right\} & =\bigcup_{m \in \mathbb{N}}\left\{\sup _{t \in\left[0, \tau_{2, n} \wedge k\right]}\|u(t)\|_{H^{s}}<m\right\} \\
& \subset \bigcup_{m \in \mathbb{N}}\left\{\tau_{2, n} \wedge k \leq \tau_{1, m}\right\} .
\end{aligned}
$$

Notice that

$$
\bigcup_{m \in \mathbb{N}}\left\{\tau_{2, n} \wedge k \leq \tau_{1, m}\right\} \subset\left\{\tau_{2, n} \wedge k \leq \tau_{1}\right\} .
$$


If $\mathbb{P}\left\{\tau_{2, n} \wedge k \leq \tau_{1}\right\}=1$ for all $n, k \in \mathbb{N}$, then we have

$$
\mathbb{P}\left\{\tau_{2} \leq \tau_{1}\right\}=\mathbb{P}\left\{\bigcap_{n \in \mathbb{N}}\left\{\tau_{2, n} \leq \tau_{1}\right\}\right\}=\mathbb{P}\left\{\bigcap_{n, k \in \mathbb{N}}\left\{\tau_{2, n} \wedge k \leq \tau_{1}\right\}\right\}=1 .
$$

To this end, we only need to prove

$$
\mathbb{P}\left\{\sup _{t \in\left[0, \tau_{2, n} \wedge k\right]}\|u(t)\|_{H^{s}}<\infty\right\}=1, \quad \forall n, k \in \mathbb{N} .
$$

Consider first $\mathbb{E} \sup _{t \in\left[0, \tau_{2, n} \wedge k\right]}\|u(t)\|_{H^{s}}^{2}$. We cannot estimate this expectation using the Itô formula directly. Indeed, the Itô formula in a Hilbert space ([18, Theorem 4.32] or [26, Theorem 2.10]) requires $\left((u-\gamma) u_{x}, u\right)_{H^{s}}$ to be welldefined and the Itô formula under a Gelfand triplet ([38, Theorem I.3.1] or [44, Theorem 4.2.5]) requires the dual product $H^{s-1}\left\langle(u-\gamma) u_{x}, u\right\rangle_{H^{s+1}}$ to be well-defined. In our case we only have $u \in H^{s}$ and $(u-\gamma) u_{x} \in H^{s-1}$ such that neither requirement is fulfilled. Therefore we utilize the mollifier operator $T_{\varepsilon}$ defined in (3.1). We first apply $T_{\varepsilon}$ to (1.8), and then use the Itô formula for $\left\|T_{\varepsilon} u\right\|_{H^{s}}^{2}=\left\|D^{s} T_{\varepsilon} u\right\|_{L^{2}}^{2}$ to deduce that for any $n, k>1$ and $t \in\left[0, \tau_{2, n} \wedge k\right]$,

$$
\begin{aligned}
\left\|T_{\varepsilon} u(t)\right\|_{H^{s}}^{2}-\left\|T_{\varepsilon} u(0)\right\|_{H^{s}}^{2}= & 2 \sum_{k=1}^{\infty} \int_{0}^{t}\left(D^{s} T_{\varepsilon} h\left(t^{\prime}, u\right) e_{k}, D^{s} T_{\varepsilon} u\right)_{L^{2}} \mathrm{~d} W_{k} \\
& -2 \int_{0}^{t}\left(D^{s} T_{\varepsilon}\left[(u-\gamma) \partial_{x} u\right], D^{s} T_{\varepsilon} u\right)_{L^{2}} \mathrm{~d} t^{\prime} \\
& -2 \int_{0}^{t}\left(D^{s} T_{\varepsilon} F(u), D^{s} T_{\varepsilon} u\right)_{L^{2}} \mathrm{~d} t^{\prime} \\
& +\int_{0}^{t} \sum_{k=1}^{\infty}\left\|D^{s} T_{\varepsilon} h\left(t^{\prime}, u\right) e_{k}\right\|_{L^{2}}^{2} \mathrm{~d} t^{\prime} \\
=: & \sum_{k=1}^{\infty} \int_{0}^{t} L_{1, k} \mathrm{~d} W_{k}+\sum_{i=2}^{4} \int_{0}^{t} L_{i} \mathrm{~d} t^{\prime} .
\end{aligned}
$$

On account of the Burkholder-Davis-Gundy inequality (2.1), for the expectation of the $H^{s}$-norm of $T_{\varepsilon} u$, we arrive at

$$
\begin{aligned}
\mathbb{E} \sup _{t \in\left[0, \tau_{2, n} \wedge k\right]}\left\|T_{\varepsilon} u(t)\right\|_{H^{s}}^{2} \leq & \mathbb{E}\left\|T_{\varepsilon} u_{0}\right\|_{H^{s}}^{2}+C \mathbb{E}\left(\int_{0}^{\tau_{2, n} \wedge k} \sum_{k=1}^{\infty}\left|L_{1, k}\right|^{2} \mathrm{~d} t\right)^{\frac{1}{2}} \\
& +\sum_{i=2}^{4} \mathbb{E} \int_{0}^{\tau_{2, n} \wedge k}\left|L_{i}\right| \mathrm{d} t
\end{aligned}
$$

We can infer from (3.4) and Assumption 2.1 that 


$$
\begin{aligned}
& \mathbb{E}\left(\int_{0}^{\tau_{2, n} \wedge k} \sum_{k=1}^{\infty}\left|L_{1, k}\right|^{2} \mathrm{~d} t\right)^{\frac{1}{2}} \\
& \quad \leq \frac{1}{2} \mathbb{E} \sup _{t \in\left[0, \tau_{2, n} \wedge k\right]}\left\|T_{\varepsilon} u\right\|_{H^{s}}^{2}+C f^{2}(n) \int_{0}^{k}\left(1+\mathbb{E}\|u\|_{H^{s}}^{2}\right) \mathrm{d} t .
\end{aligned}
$$

For $L_{2}$ and $L_{3}$, we use Lemma 3.5 to find

$$
\mathbb{E} \int_{0}^{\tau_{2, n} \wedge k}\left|L_{2}\right|+\left|L_{3}\right| \mathrm{d} t \leq C(1+n) \int_{0}^{k}\left(1+\mathbb{E}\|u\|_{H^{s}}^{2}\right) \mathrm{d} t .
$$

Similarly, it follows from the assumption (2.2) that

$$
\mathbb{E} \int_{0}^{\tau_{2, n} \wedge k}\left|L_{4}\right| \mathrm{d} t \leq C f^{2}(n) \int_{0}^{k}\left(1+\mathbb{E}\|u\|_{H^{s}}^{2}\right) \mathrm{d} t .
$$

If we combine the above estimates and use (3.4), we are led for some constant $C=C_{n}>0$ depending on $n$ to

$$
\mathbb{E} \sup _{t \in\left[0, \tau_{2, n} \wedge k\right]}\left\|T_{\varepsilon} u(t)\right\|_{H^{s}}^{2} \leq 2 \mathbb{E}\left\|u_{0}\right\|_{H^{s}}^{2}+C_{n} \int_{0}^{k}\left(1+\mathbb{E} \sup _{t^{\prime} \in\left[0, t \wedge \tau_{2, n}\right]}\left\|u\left(t^{\prime}\right)\right\|_{H^{s}}^{2}\right) \mathrm{d} t .
$$

Since the right hand side of the last estimate does not depend on $\varepsilon$, and $T_{\varepsilon} u$ tends to $u$ in $C\left([0, T] ; H^{s}\right)$ for any $T>0$ almost surely as $\varepsilon \rightarrow 0$, one can send $\varepsilon \rightarrow 0$ to obtain

$$
\mathbb{E} \sup _{t \in\left[0, \tau_{2, n} \wedge k\right]}\|u(t)\|_{H^{s}}^{2} \leq 2 \mathbb{E}\left\|u_{0}\right\|_{H^{s}}^{2}+C_{n} \int_{0}^{k}\left(1+\mathbb{E} \sup _{t^{\prime} \in\left[0, t \wedge \tau_{2, n}\right]}\left\|u\left(t^{\prime}\right)\right\|_{H^{s}}^{2}\right) \mathrm{d} t .
$$

Then Grönwall's inequality shows that for each $n, k \in \mathbb{N}$, there is a constant $C=C\left(n, k, u_{0}\right)>0$ such that

$$
\mathbb{E} \sup _{t \in\left[0, \tau_{2, n} \wedge k\right]}\|u(t)\|_{H^{s}}^{2}<C\left(n, k, u_{0}\right),
$$

which gives (4.2).

We finish the section with the proof of the blow-up criterion in Theorem (2.1).

Proof of (2.5). Let $\tau_{1, m}, \tau_{2, n}, \tau_{1}$ and $\tau_{2}$ be given in Lemma 4.1. If $u$ is the unique pathwise solution with maximal existence time $\tau^{*}$, for fixed $m, n>0$, even if $\mathbb{P}\left\{\tau_{1, m}=0\right\}$ or $\mathbb{P}\left\{\tau_{2, n}=0\right\}$ is larger than 0 , for a.e. $\omega \in \Omega$, there is $m>0$ or $n>0$ such that $\tau_{1, m}, \tau_{2, n}>0$. By continuity of $\|u(t)\|_{H^{s}}$ and the uniqueness of $u$, it is easy to check that $\tau_{1}=\tau_{2}=\tau^{*}$. Consequently, we obtain the desired blow-up criterion.

\section{Proof of Theorem 2.2: strong nonlinear noise}

To begin with, we note the following algebraic inequality. 
Lemma 5.1. Let $c, M>0$. Assume

$$
\text { either } \eta>1, a, b>0 \text { or } \eta=1, b>a>0 .
$$

There is a $C>0$ such that for all $0 \leq x \leq M y<\infty$,

$$
\frac{a(1+x) y^{2}+b(1+x)^{\eta} y^{2}}{1+y^{2}}-\frac{2 b(1+x)^{\eta} y^{4}}{\left(1+y^{2}\right)^{2}}+\frac{c(1+x)^{\eta} y^{4}}{\left(1+y^{2}\right)^{2}\left(1+\log \left(1+y^{2}\right)\right)} \leq C .
$$

Proof. Since $M y \geq x$, we have

$$
\begin{aligned}
& \frac{a(1+x) y^{2}+b(1+x)^{\eta} y^{2}}{1+y^{2}}-\frac{2 b(1+x)^{\eta} y^{4}}{\left(1+y^{2}\right)^{2}}+\frac{c(1+x)^{\eta} y^{4}}{\left(1+y^{2}\right)^{2}\left(1+\log \left(1+y^{2}\right)\right)} \\
& \leq a(1+x)+b(1+x)^{\eta}-2 b(1+x)^{\eta} \frac{\left(\frac{x}{M}\right)^{4}}{\left(1+\left(\frac{x}{M}\right)^{2}\right)^{2}}+\frac{c(1+x)^{\eta}}{\left(1+\log \left(1+\left(\frac{x}{M}\right)^{2}\right)\right)} .
\end{aligned}
$$

When $\eta>1$ and $a, b>0$ or $\eta=1$ and $b>a>0$, the latter expression tends to $-\infty$ for $x \rightarrow+\infty$, which implies the statement of the lemma.

We are now ready to prove Theorem 2.2 following [45] to large extent.

Proof of Theorem 2.2. Assume $s>5 / 2$ and let $u_{0}$ be an $H^{s}$-valued $\mathcal{F}_{0}$-measurable random variable with $\mathbb{E}\left\|u_{0}\right\|_{H^{s}}^{2}<\infty$. Let $h(t, u)=h(u)=a$ $\left(1+\|u\|_{W^{1, \infty}}\right)^{\theta} u$ with $\theta \geq 1 / 2$ and $a \neq 0$.

For $r>3 / 2$, the embedding $H^{r} \hookrightarrow W^{1, \infty}$ implies that we have for any $u, v \in H^{r}$ the estimate

$$
\sup _{\|u\|_{H^{r}},\|v\|_{H^{r}} \leq N}\left\{\mathbf{1}_{\{u \neq v\}} \frac{\|h(u)-h(v)\|_{H^{r}}}{\|u-v\|_{H^{r}}}\right\} \leq q(N), \quad N \geq 1 .
$$

This means that one can establish the pathwise uniqueness for (1.10) in $H^{r}$ with $r>3 / 2$. Hence, in the same way as proving Theorem 2.1, one can show that (1.10) admits a unique pathwise solution $u$ in $H^{s}$ with $s>5 / 2$ and maximal existence time $\tau^{*}$. We recall the definition of the mollifier $T_{\varepsilon}$ from Sect. 3 and define

$$
\tau_{m}=\inf \left\{t \geq 0:\|u(t)\|_{H^{s}} \geq m\right\} .
$$

Applying the Itô formula to $\left\|T_{\varepsilon} u(t)\right\|_{H^{s}}^{2}$ gives

$$
\begin{aligned}
\mathrm{d}\left\|T_{\varepsilon} u\right\|_{H^{s}}^{2}= & 2 a\left(1+\|u\|_{W^{1, \infty}}\right)^{\theta}\left(T_{\varepsilon} u, T_{\varepsilon} u\right)_{H^{s}} \mathrm{~d} W-2\left(T_{\varepsilon}\left[(u-\gamma) u_{x}\right], T_{\varepsilon} u\right)_{H^{s}} \mathrm{~d} t \\
& -2\left(T_{\varepsilon} F(u), T_{\varepsilon} u\right)_{H^{s}} \mathrm{~d} t+a^{2}\left(1+\|u\|_{W^{1, \infty}}\right)^{2 \theta}\left\|T_{\varepsilon} u\right\|_{H^{s}}^{2} \mathrm{~d} t .
\end{aligned}
$$

Again, using Itô formula to $\log \left(1+\left\|T_{\varepsilon} u\right\|_{H^{s}}^{2}\right)$ yields

$$
\begin{aligned}
\mathrm{d} \log ( & \left.1+\left\|T_{\varepsilon} u\right\|_{H^{s}}^{2}\right) \\
= & \frac{2 a\left(1+\|u\|_{W^{1, \infty}}\right)^{\theta}}{1+\left\|T_{\varepsilon} u\right\|_{H^{s}}^{2}}\left(T_{\varepsilon} u, T_{\varepsilon} u\right)_{H^{s}} \mathrm{~d} W \\
& -\frac{1}{1+\left\|T_{\varepsilon} u\right\|_{H^{s}}^{2}}\left\{2\left(T_{\varepsilon}\left[(u-\gamma) u_{x}\right], T_{\varepsilon} u\right)_{H^{s}}+2\left(T_{\varepsilon} F(u), T_{\varepsilon} u\right)_{H^{s}}\right\} \mathrm{d} t \\
& +\frac{a^{2}\left(1+\|u\|_{W^{1, \infty}}\right)^{2 \theta}}{1+\left\|T_{\varepsilon} u\right\|_{H^{s}}^{2}}\left\|T_{\varepsilon} u\right\|_{H^{s}}^{2} \mathrm{~d} t-2 \frac{a^{2}\left(1+\|u\|_{W^{1, \infty}}\right)^{2 \theta}}{\left(1+\left\|T_{\varepsilon} u\right\|_{H^{s}}^{2}\right)^{2}}\left\|T_{\varepsilon} u\right\|_{H^{s}}^{4} \mathrm{~d} t .
\end{aligned}
$$


Lemma 3.5 and (3.4) imply that there is a $Q=Q\left(s, c_{0}, \gamma\right)>0$ such that for any $t>0$ we have

$\mathbb{E} \log \left(1+\left\|T_{\varepsilon} u\left(t \wedge \tau_{m}\right)\right\|_{H^{s}}^{2}\right)-\mathbb{E} \log \left(1+\left\|T_{\varepsilon} u_{0}\right\|_{H^{s}}^{2}\right)$

$$
\begin{aligned}
= & \mathbb{E} \int_{0}^{t \wedge \tau_{m}} \frac{1}{1+\left\|T_{\varepsilon} u\right\|_{H^{s}}^{2}}\left\{-2\left(T_{\varepsilon}\left[(u-\gamma) u_{x}\right], T_{\varepsilon} u\right)_{H^{s}}\right. \\
& \left.-2\left(T_{\varepsilon} F(u), T_{\varepsilon} u\right)_{H^{s}}\right\} \mathrm{d} t^{\prime} \\
& +\mathbb{E} \int_{0}^{t \wedge \tau_{m}} \frac{1}{1+\left\|T_{\varepsilon} u\right\|_{H^{s}}^{2}} a^{2}\left(1+\|u\|_{W^{1, \infty}}\right)^{2 \theta}\left\|T_{\varepsilon} u\right\|_{H^{s}}^{2} \mathrm{~d} t^{\prime} \\
& -\mathbb{E} \int_{0}^{t \wedge \tau_{m}} \frac{2}{\left(1+\left\|T_{\varepsilon} u\right\|_{H^{s}}^{2}\right)^{2}} a^{2}\left(1+\|u\|_{W^{1, \infty}}\right)^{2 \theta}\left\|T_{\varepsilon} u\right\|_{H^{s}}^{4} \mathrm{~d} t^{\prime} \\
\leq & \mathbb{E} \int_{0}^{t \wedge \tau_{m}}\left[\frac { 1 } { 1 + \| T _ { \varepsilon } u \| _ { H ^ { s } } ^ { 2 } } \left\{2 Q\left(1+\|u\|_{W^{1, \infty}}\right)\|u\|_{H^{s}}^{2}+a^{2}\left(1+\|u\|_{W^{1, \infty}}\right)^{2 \theta}\right.\right. \\
& \left.\left.\left\|T_{\varepsilon} u\right\|_{H^{s}}^{2}\right\}\right] \mathrm{d} t^{\prime} \\
-\mathbb{E} & \int_{0}^{t \wedge \tau_{m}} \frac{1}{\left(1+\left\|T_{\varepsilon} u\right\|_{H^{s}}^{2}\right)^{2}} 2 a^{2}\left(1+\|u\|_{W^{1, \infty}}\right)^{2 \theta}\left\|T_{\varepsilon} u\right\|_{H^{s}}^{4} \mathrm{~d} t^{\prime} .
\end{aligned}
$$

Notice that for any $T>0, T_{\varepsilon} u$ tends to $u$ in $C\left([0, T] ; H^{s}\right)$ almost surely as $\varepsilon \rightarrow 0$. Then, by (3.4) and the dominated convergence theorem, the last estimate leads to

$$
\begin{gathered}
\mathbb{E} \log \left(1+\left\|u\left(t \wedge \tau_{m}\right)\right\|_{H^{s}}^{2}\right)-\mathbb{E} \log \left(1+\left\|u_{0}\right\|_{H^{s}}^{2}\right) \\
=\lim _{\varepsilon \rightarrow 0}\left(\mathbb{E} \log \left(\left(1+\left\|T_{\varepsilon} u\left(t \wedge \tau_{m}\right)\right\|_{H^{s}}^{2}\right)\right)-\mathbb{E} \log \left(\left(1+\left\|T_{\varepsilon} u_{0}\right\|_{H^{s}}^{2}\right)\right)\right) \\
\leq \lim _{\varepsilon \rightarrow 0} \mathbb{E} \int_{0}^{t \wedge \tau_{m}} \frac{1}{1+\left\|T_{\varepsilon} u\right\|_{H^{s}}^{2}}\left\{2 Q\left(1+\|u\|_{W^{1, \infty}}\right)\|u\|_{H^{s}}^{2}\right. \\
\left.+a^{2}\left(1+\|u\|_{W^{1, \infty}}\right)^{2 \theta}\left\|T_{\varepsilon} u\right\|_{H^{s}}^{2}\right\} \mathrm{d} t^{\prime} \\
-\lim _{\varepsilon \rightarrow 0} \mathbb{E} \int_{0}^{t \wedge \tau_{m}} \frac{1}{\left(1+\left\|T_{\varepsilon} u\right\|_{H^{s}}^{2}\right)^{2}} 2 a^{2}\left(1+\|u\|_{W^{1, \infty}}\right)^{2 \theta}\left\|T_{\varepsilon} u\right\|_{H^{s}}^{4} \mathrm{~d} t^{\prime} \\
=\mathbb{E} \int_{0}^{t \wedge \tau_{m}} \frac{2 Q\left(1+\|u\|_{W^{1, \infty}}\right)\|u\|_{H^{s}}^{2}+a^{2}\left(1+\|u\|_{W^{1, \infty}}\right)^{2 \theta}\|u\|_{H^{s}}^{2}}{1+\|u\|_{H^{s}}^{2}} \mathrm{~d} t^{\prime} \\
\left.-\mathbb{E} \int_{0}^{t \wedge \tau_{m}} \frac{2 a^{2}\left(1+\|u\|_{W^{1, \infty}}\right)^{2 \theta}\|u\|_{H^{s}}^{4}}{\left(1+\|u\|_{H^{s}}^{2}\right.}\right)^{2} .
\end{gathered}
$$

Since we have assumed (2.6), Lemma 5.1 immediately shows that there are constants $K_{1}, K_{2}>0$ such that

$$
\begin{aligned}
& \mathbb{E} \log \left(1+\left\|u\left(t \wedge \tau_{m}\right)\right\|_{H^{s}}^{2}\right)-\mathbb{E} \log \left(1+\left\|u_{0}\right\|_{H^{s}}^{2}\right) \\
& \quad \leq \mathbb{E} \int_{0}^{t \wedge \tau_{m}} K_{1}-K_{2} \frac{a^{2}\left(1+\|u\|_{W^{1, \infty}}\right)^{2 \theta}\|u\|_{H^{s}}^{4}}{\left(1+\|u\|_{H^{s}}^{2}\right)^{2}\left(1+\log \left(1+\|u\|_{H^{s}}^{2}\right)\right)} \mathrm{d} t^{\prime},
\end{aligned}
$$


which means that for some $C\left(u_{0}, K_{1}, K_{2}, t\right)>0$,

$$
\mathbb{E} \int_{0}^{t \wedge \tau_{m}} \frac{a^{2}\left(1+\|u\|_{W^{1, \infty}}\right)^{2 \theta}\|u\|_{H^{s}}^{4}}{\left(1+\|u\|_{H^{s}}^{2}\right)^{2}\left(1+\log \left(1+\|u\|_{H^{s}}^{2}\right)\right)} \mathrm{d} t^{\prime} \leq C\left(u_{0}, K_{1}, K_{2}, t\right)<\infty,
$$

and

$$
\begin{aligned}
& \mathbb{E} \int_{0}^{t \wedge \tau_{m}}\left|K_{1}-K_{2} \frac{a^{2}\left(1+\|u\|_{W^{1, \infty}}\right)^{2 \theta}\|u\|_{H^{s}}^{4}}{\left(1+\|u\|_{H^{s}}^{2}\right)^{2}\left(1+\log \left(1+\|u\|_{H^{s}}^{2}\right)\right)}\right| \mathrm{d} t^{\prime} \\
& \quad \leq C\left(u_{0}, K_{1}, K_{2}, t\right)<\infty .
\end{aligned}
$$

Next, we notice that there is a function $\delta:[0, \infty) \rightarrow[0, \infty)$ with $\delta(\varepsilon) \rightarrow 0$ when $\varepsilon \rightarrow 0$ such that

$$
\begin{aligned}
& \frac{2 Q\left(1+\|u\|_{W^{1, \infty}}\right)\|u\|_{H^{s}}^{2}+a^{2}\left(1+\|u\|_{W^{1, \infty}}\right)^{2 \theta}\left\|T_{\varepsilon} u\right\|_{H^{s}}^{2}}{1+\left\|T_{\varepsilon} u\right\|_{H^{s}}^{2}} \\
& -\frac{2 a^{2}\left(1+\|u\|_{W^{1, \infty}}\right)^{2 \theta}\left\|T_{\varepsilon} u\right\|_{H^{s}}^{4}}{\left(1+\left\|T_{\varepsilon} u\right\|_{H^{s}}^{2}\right)^{2}} \\
& \leq \frac{2 Q\left(1+\|u\|_{W^{1, \infty}}\right)\|u\|_{H^{s}}^{2}+a^{2}\left(1+\|u\|_{W^{1, \infty}}\right)^{2 \theta}\|u\|_{H^{s}}^{2}}{1+\|u\|_{H^{s}}^{2}} \\
& -\frac{2 a^{2}\left(1+\|u\|_{W^{1, \infty}}\right)^{2 \theta}\|u\|_{H^{s}}^{4}}{\left(1+\|u\|_{H^{s}}^{2}\right)^{2}}+\delta(\varepsilon)
\end{aligned}
$$

holds. Therefore, for any $T>0$, by using Lemma 5.1, the Burkholder-DavisGundy inequality (2.1) and (5.2), we find that

$$
\begin{aligned}
& \mathbb{E} \sup _{t \in\left[0, T \wedge \tau_{m}\right]} \log \left(1+\left\|T_{\varepsilon} u\right\|_{H^{s}}^{2}\right)-\mathbb{E} \log \left(1+\left\|T_{\varepsilon} u_{0}\right\|_{H^{s}}^{2}\right) \\
& \leq C \mathbb{E}\left(\int_{0}^{T \wedge \tau_{m}} \frac{a^{2}\left(1+\|u\|_{W^{1, \infty}}\right)^{2 \theta}\left\|T_{\varepsilon} u\right\|_{H^{s}}^{4}}{\left(1+\left\|T_{\varepsilon} u\right\|_{H^{s}}^{2}\right)^{2}} \mathrm{~d} t\right)^{\frac{1}{2}} \\
&+\mathbb{E} \int_{0}^{T \wedge \tau_{m}}\left|K_{1}-K_{2} \frac{a^{2}\left(1+\|u\|_{W^{1, \infty}}\right)^{2 \theta}\|u\|_{H^{s}}^{4}}{\left(1+\|u\|_{H^{s}}^{2}\right)^{2}\left(1+\log \left(1+\|u\|_{H^{s}}^{2}\right)\right)}+\delta(\varepsilon)\right| \mathrm{d} t \\
& \leq \frac{1}{2} \mathbb{E} \sup _{t \in\left[0, T \wedge \tau_{m}\right]}\left(1+\log \left(1+\left\|T_{\varepsilon} u\right\|_{H^{s}}^{2}\right)\right) \\
&+C \mathbb{E} \int_{0}^{T \wedge \tau_{m}} \frac{a^{2}\left(1+\|u\|_{W^{1, \infty}}\right)^{2 \theta}\left\|T_{\varepsilon} u\right\|_{H^{s}}^{4}}{\left(1+\left\|T_{\varepsilon} u\right\|_{H^{s}}^{2}\right)^{2}\left(1+\log \left(1+\left\|T_{\varepsilon} u\right\|_{H^{s}}^{2}\right)\right)} \mathrm{d} t \\
&+K_{1} T+\mathbb{E} \int_{0}^{T \wedge \tau_{m}} K_{2} \frac{a^{2}\left(1+\|u\|_{W^{1, \infty}}\right)^{2 \theta}\|u\|_{H^{s}}^{4}}{\left(1+\|u\|_{H^{s}}^{2}\right)^{2}\left(1+\log \left(1+\|u\|_{H^{s}}^{2}\right)\right)} \mathrm{d} t+\delta(\varepsilon) T \\
& \leq \frac{1}{2} \mathbb{E} \sup _{t \in\left[0, T \wedge \tau_{m}\right]}\left(1+\log \left(1+\left\|T_{\varepsilon} u\right\|_{H^{s}}^{2}\right)\right) \\
&+C \mathbb{E} \int_{0}^{T \wedge \tau_{m}} \frac{a^{2}\left(1+\|u\|_{W^{1, \infty}}\right)^{2 \theta}\left\|T_{\varepsilon} u\right\|_{H^{s}}^{4}}{\left(1+\left\|T_{\varepsilon} u\right\|_{H^{s}}^{2}\right)^{2}\left(1+\log \left(1+\left\|T_{\varepsilon} u\right\|_{H^{s}}^{2}\right)\right)} \mathrm{d} t
\end{aligned}
$$




$$
+C\left(u_{0}, K_{1}, K_{2}, T\right)+\delta(\varepsilon) T .
$$

Thus, we use the dominated convergence theorem, Fatou's lemma and (5.1) to obtain finally

$$
\mathbb{E} \sup _{t \in\left[0, T \wedge \tau_{m}\right]} \log \left(1+\|u\|_{H^{s}}^{2}\right) \leq C\left(u_{0}, K_{1}, K_{2}, T\right) .
$$

Since $\log (1+x)$ is continuous and increasing for $x>0$, we have that for any $m \geq 1$,

$$
\begin{aligned}
& \mathbb{P}\left\{\tau^{*}<T\right\} \leq \mathbb{P}\left\{\tau_{m}<T\right\} \leq \mathbb{P}\left\{\sup _{t \in[0, T]} \log \left(1+\|u\|_{H^{s}}^{2}\right) \geq \log \left(1+m^{2}\right)\right\} \\
& \quad \leq \frac{C\left(u_{0}, K_{1}, K_{2}, T\right)}{\log \left(1+m^{2}\right)}
\end{aligned}
$$

Letting $m \rightarrow \infty$ forces $\mathbb{P}\left\{\tau^{*}<T\right\}=0$ for any $T>0$, which means $\mathbb{P}\left\{\tau^{*}=\right.$ $\infty\}=1$.

\section{Proofs of Theorems 2.3-2.7: non-autonomous linear noise case}

In this section, we study (1.12) with linear noise. Depending on the strength of the noise in (1.12), we provide either the global existence of pathewise solutions or the precise blow-up scenarios for the maximal pathwise solution. As discussed in Remark 2.6, we rely on the Girsanov-type transform

$$
v=\frac{1}{\beta(\omega, t)} u, \quad \beta(\omega, t)=\mathrm{e}^{\int_{0}^{t} b\left(t^{\prime}\right) \mathrm{d} W_{t^{\prime}}-\int_{0}^{t} \frac{b^{2}\left(t^{\prime}\right)}{2} \mathrm{~d} t^{\prime}} .
$$

We first collect some properties of $v$.

Proposition 6.1. Let $s>3 / 2, \alpha=1$ and $h(t, u)=b(t) u$ such that $b(t)$ satisfies Assumption 2.2. Let $\mathcal{S}=\left(\Omega, \mathcal{F}, \mathbb{P},\left\{\mathcal{F}_{t}\right\}_{t \geq 0}, W\right)$ be fixed in advance. If $u_{0}(\omega, x)$ is an $H^{s}$-valued $\mathcal{F}_{0}$ measurable random variable with $\mathbb{E}\left\|u_{0}\right\|_{H^{s}}^{2}<\infty$ and $\left(u, \tau^{*}\right)$ is the corresponding unique maximal solution to (1.11), then for any $c_{0}, \gamma \in \mathbb{R}$ and for $t \in\left[0, \tau^{*}\right)$, the process $v$ defined by (6.1) solves the following problem on $\mathbb{T}$ almost surely,

$$
\left\{\begin{array}{l}
v_{t}+\beta v v_{x}-\gamma v_{x}+\beta\left(1-\partial_{x x}^{2}\right)^{-1} \partial_{x}\left(v^{2}+\frac{1}{2} v_{x}^{2}\right)+\left(c_{0}+\gamma\right)\left(1-\partial_{x x}^{2}\right)^{-1} \partial_{x} v=0, \\
v(\omega, 0, x)=u_{0}(\omega, x) .
\end{array}\right.
$$

Moreover, we have $v \in C\left(\left[0, \tau^{*}\right) ; H^{s}\right) \cap C^{1}\left(\left[0, \tau^{*}\right) ; H^{s-1}\right) \mathbb{P}-$ a.s. and, if $s>3$, then it holds

$$
\mathbb{P}\left\{\|v(t)\|_{H^{1}}=\left\|u_{0}\right\|_{H^{1}} \quad \text { for all } t \geq 0\right\}=1
$$


Proof. Since $b(t)$ satisfies Assumption 2.2, $h(t, u)=b(t) u$ satisfies Assumption 2.1. Consequently, Theorem 2.1 implies that (1.11) (that is (1.8) with $h(t, u)=$ $b(t) u)$ has a unique maximal solution $\left(u, \tau^{*}\right)$.

A direct computation with the Itô formula yields

$$
\mathrm{d} \frac{1}{\beta}=-b(t) \frac{1}{\beta} \mathrm{d} W+b^{2}(t) \frac{1}{\beta} \mathrm{d} t .
$$

Therefore we arrive at

$$
\begin{aligned}
\mathrm{d} v= & \frac{1}{\beta}\left[-\left[(u-\gamma) \partial_{x} u+F(u)\right] \mathrm{d} t+b(t) u \mathrm{~d} W\right] \\
& \quad+u\left[-b(t) \frac{1}{\beta} \mathrm{d} W+b^{2}(t) \frac{1}{\beta} \mathrm{d} t\right]-b^{2}(t) \frac{1}{\beta} u \mathrm{~d} t \\
= & \frac{1}{\beta}\left[-\left((u-\gamma) \partial_{x} u+F(u)\right) \mathrm{d} t\right] \\
= & \left\{-\beta v v_{x}+\gamma v_{x}-\beta\left(1-\partial_{x x}^{2}\right)^{-1} \partial_{x}\left(v^{2}+\frac{1}{2} v_{x}^{2}\right)-\left(c_{0}+\gamma\right)\left(1-\partial_{x x}^{2}\right)^{-1} v_{x}\right\} \mathrm{d} t,
\end{aligned}
$$

which is $(6.2)_{1}$. Since $v(0)=u_{0}(\omega, x)$, we see that $v$ satisfies (6.2). Moreover, Theorem 2.1 implies $u \in C\left(\left[0, \tau^{*}\right) ; H^{s}\right) \mathbb{P}-$ a.s., so is $v$. Besides, from Lemma 3.4 and $(6.2)_{1}$, we see that for a.e. $\omega \in \Omega, v_{t}=\gamma v_{x}-\beta v v_{x}-\beta\left(F_{1}(v)+F_{2}(v)\right)-$ $F_{3}(v) \in C\left(\left[0, \tau^{*}\right) ; H^{s-1}\right)$. Hence $v \in C^{1}\left(\left[0, \tau^{*}\right) ; H^{s-1}\right) \mathbb{P}-$ a.s.

Notice that if $s>3,(6.2)_{1}$ is equivalent to

$$
v_{t}-v_{x x t}+c_{0} v_{x}+\gamma v_{x x x}+3 \beta v v_{x}=2 \beta v_{x} v_{x x}+\beta v v_{x x x} .
$$

Multiplying both sides of (6.5) by $v$ and then integrating the resulting equation on $x \in \mathbb{T}$, we see that for a.e. $\omega \in \Omega$ and for all $t>0$,

$$
\frac{\mathrm{d}}{\mathrm{d} t} \int_{\mathbb{T}}\left(v^{2}+v_{x}^{2}\right) \mathrm{d} x=0
$$

which implies (6.3).

\subsection{Theorem 2.3: global existence for weak noise I}

Now we prove the first global existence result, which is motivated by $[29,46-$ $48]$.

Proof of Theorem 2.3. To begin with, we apply the operator $D^{s}$ to $(6.4)$, multiply both sides of the resulting equation by $D^{s} v$ and integrate over $\mathbb{T}$ to obtain for a.e. $\omega \in \Omega$

$$
\begin{aligned}
\frac{1}{2} \frac{\mathrm{d}}{\mathrm{d} t}\|v(t)\|_{H^{s}}^{2}=\gamma \int_{\mathbb{T}} D^{s} v \cdot D^{s} v_{x} \mathrm{~d} x-\beta(\omega, t) \int_{\mathbb{T}} D^{s} v \cdot D^{s}\left[v v_{x}\right] \mathrm{d} x \\
-\beta(\omega, t) \int_{\mathbb{T}} D^{s} v \cdot D^{s} F(v) \mathrm{d} x \\
=-\beta(\omega, t) \int_{\mathbb{T}} D^{s} v \cdot D^{s}\left[v v_{x}\right] \mathrm{d} x \\
-\beta(\omega, t) \int_{\mathbb{T}} D^{s} v \cdot D^{s} F(v) \mathrm{d} x .
\end{aligned}
$$


Using Lemma 3.2, integration by parts and Lemma 3.4, we conclude that there is a $C=C(s)>1$ such that for a.e. $\omega \in \Omega$ we have

$$
\frac{\mathrm{d}}{\mathrm{d} t}\|v(t)\|_{H^{s}}^{2} \leq C \beta(t)\|v\|_{W^{1, \infty}}\|v\|_{H^{s}}^{2},
$$

where $\beta$ is given in (6.1) (If necessary, $T_{\varepsilon}$ can be used as in Lemma 4.1). Then

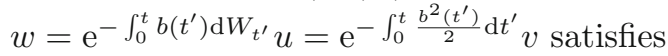

$$
\begin{aligned}
& \frac{\mathrm{d}}{\mathrm{d} t}\|w(t)\|_{H^{s}}+\frac{b^{2}(t)}{2}\|w(t)\|_{H^{s}} \\
& \quad \leq C \alpha(\omega, t)\|w(t)\|_{W^{1, \infty}}\|w(t)\|_{H^{s}}, \quad \alpha(\omega, t)=\mathrm{e}^{\int_{0}^{t} b\left(t^{\prime}\right) \mathrm{d} W_{t^{\prime}}} .
\end{aligned}
$$

Let $R>1$ and $\lambda_{1}>2$. Assume $\left\|u_{0}\right\|_{H^{s}}<\frac{b_{*}}{C K \lambda_{1} R}<\frac{b_{*}}{C K \lambda_{1}}$ almost surely and define

$$
\tau_{1}=\inf \left\{t>0: \alpha(\omega, t)\|w\|_{W^{1, \infty}}=\|u\|_{W^{1, \infty}}>\frac{b^{2}(t)}{C \lambda_{1}}\right\} .
$$

Then it follows from the embedding $\|u(0)\|_{W^{1, \infty}} \leq K\|u(0)\|_{H^{s}}<\frac{b_{*}}{C \lambda_{1}}$ that $\mathbb{P}\left\{\tau_{1}>0\right\}=1$, and for $t \in\left[0, \tau_{1}\right)$,

$$
\frac{\mathrm{d}}{\mathrm{d} t}\|w(t)\|_{H^{s}}+\frac{\left(\lambda_{1}-2\right) b^{2}(t)}{2 \lambda_{1}}\|w(t)\|_{H^{s}} \leq 0 .
$$

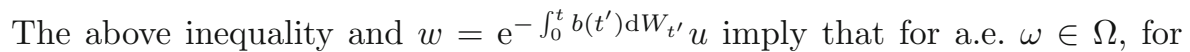
any $\lambda_{2}>\frac{2 \lambda_{1}}{\lambda_{1}-2}$ and for $t \in\left[0, \tau_{1}\right)$,

$$
\begin{aligned}
\|u(t)\|_{H^{s}} & \leq\left\|w_{0}\right\|_{H^{s}} \mathrm{e}^{\int_{0}^{t} b\left(t^{\prime}\right) \mathrm{d} W_{t^{\prime}}-\int_{0}^{t} \frac{\left(\lambda_{1}-2\right) b^{2}\left(t^{\prime}\right)}{2 \lambda_{1}} \mathrm{~d} t^{\prime}} \\
& =\left\|u_{0}\right\|_{H^{s}} \mathrm{e}^{\int_{0}^{t} b\left(t^{\prime}\right) \mathrm{d} W_{t^{\prime}}-\int_{0}^{t} \frac{b^{2}\left(t^{\prime}\right)}{\lambda_{2}} \mathrm{~d} t^{\prime}} \mathrm{e}^{-\frac{\left(\left(\lambda_{1}-2\right) \lambda_{2}-2 \lambda_{1}\right)}{2 \lambda_{1} \lambda 2} \int_{0}^{t} b^{2}\left(t^{\prime}\right) \mathrm{d} t^{\prime}} .
\end{aligned}
$$

Define the stopping time

$$
\tau_{2}=\inf \left\{t>0: \mathrm{e}^{\int_{0}^{t} b\left(t^{\prime}\right) \mathrm{d} W_{t^{\prime}}-\int_{0}^{t} \frac{b^{2}\left(t^{\prime}\right)}{\lambda_{2}} \mathrm{~d} t^{\prime}}>R\right\} .
$$

Notice that $\mathbb{P}\left\{\tau_{2}>0\right\}=1$. From (6.7), we have that almost surely

$$
\begin{aligned}
\|u(t)\|_{H^{s}} & <\frac{b_{*}}{C K \lambda_{1} R} \times R \times \mathrm{e}^{-\frac{\left(\left(\lambda_{1}-2\right) \lambda_{2}-2 \lambda_{1}\right)}{2 \lambda_{1} \lambda 2} \int_{0}^{t} b^{2}\left(t^{\prime}\right) \mathrm{d} t^{\prime}} \\
& =\frac{b_{*}}{C K \lambda_{1}} \mathrm{e}^{-\frac{\left(\left(\lambda_{1}-2\right) \lambda_{2}-2 \lambda_{1}\right)}{2 \lambda_{1} \lambda 2} \int_{0}^{t} b^{2}\left(t^{\prime}\right) \mathrm{d} t^{\prime}} \leq \frac{b_{*}}{C K \lambda_{1}}, \quad t \in\left[0, \tau_{1} \wedge \tau_{2}\right) .
\end{aligned}
$$

By Assumption 2.2, (6.9) and (6.6), we find that on $\left[0, \tau_{1} \wedge \tau_{2}\right)$,

$$
\|u(t)\|_{W^{1, \infty}} \leq K\|u(t)\|_{H^{s}} \leq \frac{b_{*}}{C \lambda_{1}} \leq \frac{b^{2}(t)}{C \lambda_{1}} \mathbb{P}-\text { a.s. },
$$

which means

$$
\mathbb{P}\left\{\tau_{1} \geq \tau_{2}\right\}=1
$$

Therefore it follows from (6.9) that

$\mathbb{P}\left\{\|u(t)\|_{H^{s}}<\frac{b_{*}}{C K \lambda_{1}} \mathrm{e}^{-\frac{\left(\left(\lambda_{1}-2\right) \lambda_{2}-2 \lambda_{1}\right)}{2 \lambda_{1} \lambda^{2}} \int_{0}^{t} b^{2}\left(t^{\prime}\right) \mathrm{d} t^{\prime}}\right.$ for all $\left.t>0\right\} \geq \mathbb{P}\left\{\tau_{2}=\infty\right\}$. 
We apply (ii) in Lemma 3.7 to find that

$$
\mathbb{P}\left\{\tau_{2}=\infty\right\}>1-\left(\frac{1}{R}\right)^{2 / \lambda_{2}},
$$

which completes the proof.

\subsection{Theorem 2.4: global existence for weak noise II}

Let $\beta(\omega, t)$ be given as in (6.1). With Proposition 6.1 at hand, we can proceed to prove Theorem 2.4. We see that for a.e. $\omega \in \Omega$, the transform $v(\omega, t, x)$ solves (6.2) on $\left[0, \tau^{*}\right)$. Moreover, since $H^{s} \hookrightarrow C^{2}$ for $s>3$, we have $v, v_{x} \in$ $C^{1}\left(\left[0, \tau^{*}\right) \times \mathbb{T}\right)$. Then for a.e. $\omega \in \Omega$, for any $x \in \mathbb{T}$ and $c_{0}, \gamma \in \mathbb{R}$, the problem

$$
\left\{\begin{array}{l}
\frac{\mathrm{d} q(\omega, t, x)}{\mathrm{d} t}=\beta(\omega, t) v(\omega, t, q(\omega, t, x))-\gamma, \quad t \in\left[0, \tau^{*}\right), \\
q(\omega, 0, x)=x, \quad x \in \mathbb{T}
\end{array}\right.
$$

has a unique solution $q(\omega, t, x)$ such that $q(\omega, t, x) \in C^{1}\left(\left[0, \tau^{*}\right) \times \mathbb{T}\right)$ for a.e $\omega \in \Omega$. Moreover, differentiating (6.11) with respect to $x$ yields that for a.e. $\omega \in \Omega$,

$$
\left\{\begin{array}{l}
\frac{d q_{x}(\omega, t, x)}{d t}=\beta(\omega, t) v_{x}(\omega, t, q) q_{x}, \quad t \in\left[0, \tau^{*}\right), \\
q_{x}(\omega, 0, x)=1, \quad x \in \mathbb{T} .
\end{array}\right.
$$

For a.e. $\omega \in \Omega$, we solve the above equation to obtain

$$
q_{x}(\omega, t, x)=\exp \left(\int_{0}^{t} \beta\left(\omega, t^{\prime}\right) v_{x}\left(\omega, t^{\prime}, q\left(\omega, t^{\prime}, x\right)\right) \mathrm{d} t^{\prime}\right) .
$$

Thus for a.e. $\omega \in \Omega, q_{x}>0,(t, x) \in\left[0, \tau^{*}\right) \times \mathbb{T}$. On the other hand, if $v$ solves (6.2) (or equivalently (6.5)) $\mathbb{P}-$ a.s., then the momentum variable $V=v-v_{x x}$ satisfies

$$
V_{t}+c_{0} v_{x}+\beta v V_{x}+2 \beta V v_{x}+\gamma v_{x x x}=0 \mathbb{P}-a . s .
$$

Particularly, if $c_{0}+\gamma=0$, (6.12) becomes

$V_{t}+c_{0} v_{x}+\beta v V_{x}+2 \beta V v_{x}+\gamma v_{x x x}=V_{t}-\gamma V_{x}+\beta v V_{x}+2 \beta V v_{x}=0 \mathbb{P}-$ a.s. which means

$$
\frac{\mathrm{d}}{\mathrm{d} t}\left[V(\omega, t, q(\omega, t, x)) q_{x}^{2}(\omega, t, x)\right]=q_{x}^{2}\left[V_{t}+\beta v V_{x}-\gamma V_{x}+2 \beta V v_{x}\right]=0 \mathbb{P}-\text { a.s. }
$$

This, and $q_{x}(\omega, 0, x)=1$ imply that

$$
V(\omega, t, q(\omega, t, x)) q_{x}^{2}(\omega, t, x)=V_{0}(\omega, x) .
$$

Consequently, we have $\operatorname{sign}(V)=\operatorname{sign}\left(V_{0}\right)$. Besides, since $v=G_{\mathbb{T}} * V$ with $G_{\mathbb{T}}>0$ given in $(1.7)$, we have $\operatorname{sign}(v)=\operatorname{sign}(V)$. Summarizing the above analysis, we have the following result:

Lemma 6.1. Assume $c_{0}+\gamma=0$ and $s>3$. Let $V_{0}(\omega, x)=\left(1-\partial_{x x}^{2}\right) u_{0}(\omega, x)$ and $V(\omega, t, x)=v(\omega, t, x)-v_{x x}(\omega, t, x)$, where $v(\omega, t, x)$ solves $(6.2)$ on $\left[0, \tau^{*}\right)$ $\mathbb{P}$ - a.s. Then for a.e. $\omega \in \Omega$,

$$
\operatorname{sign}(v)=\operatorname{sign}(V)=\operatorname{sign}\left(V_{0}\right), \quad(t, x) \in\left[0, \tau^{*}\right) \times \mathbb{T} .
$$


The next step is to control $\|u(\omega, t)\|_{W^{1, \infty}}$. In combination with (2.5), we will then directly verify Theorem 2.4 .

Lemma 6.2. Let all the conditions as in the statement of Proposition 6.1 hold true. Let $V$ and $V_{0}$ be defined in Lemma 6.1. If additionally we have $c_{0}+\gamma=0$ and

$$
\mathbb{P}\left\{V_{0}(\omega, x)>0, \quad \forall x \in \mathbb{T}\right\}=p, \quad \mathbb{P}\left\{V_{0}(\omega, x)<0, \quad \forall x \in \mathbb{T}\right\}=q,
$$

for some $p, q \in[0,1]$, then the maximal solution $\left(u, \tau^{*}\right)$ of (1.12) satisfies

$$
\mathbb{P}\left\{\left\|u_{x}(\omega, t)\right\|_{L^{\infty}} \leq\|u(\omega, t)\|_{L^{\infty}} \lesssim \beta(\omega, t)\left\|u_{0}\right\|_{H^{1}}, \quad \forall t \in\left[0, \tau^{*}\right)\right\} \geq p+q .
$$

Proof. Using (1.7), one can derive (see [48]) that for a.e. $\omega \in \Omega$, and for all $(t, x) \in\left[0, \tau^{*}\right) \times \mathbb{T}$,

$$
\begin{aligned}
& {\left[v+v_{x}\right](\omega, t, x)=\frac{1}{2 \sinh (\pi)} \int_{0}^{2 \pi} \mathrm{e}^{\left(x-y-2 \pi\left[\frac{x-y}{2 \pi}\right]-\pi\right)} V(\omega, t, y) \mathrm{d} y} \\
& {\left[v-v_{x}\right](\omega, t, x)=\frac{1}{2 \sinh (\pi)} \int_{0}^{2 \pi} \mathrm{e}^{\left(y-x+2 \pi\left[\frac{x-y}{2 \pi}\right]+\pi\right)} V(\omega, t, y) \mathrm{d} y}
\end{aligned}
$$

Then one can employ (6.13), (6.14) and Lemma 6.1 to obtain that for a.e. $\omega \in \Omega$ and for all $(t, x) \in\left[0, \tau^{*}\right) \times \mathbb{T}$,

$$
\begin{cases}-v(\omega, t, x) \leq v_{x}(\omega, t, x) \leq v(\omega, t, x), & \text { if } V_{0}(\omega, x)=\left(1-\partial_{x x}^{2}\right) u_{0}(\omega, x)>0, \\ v(\omega, t, x) \leq v_{x}(\omega, t, x) \leq-v(\omega, t, x), & \text { if } V_{0}(\omega, x)=\left(1-\partial_{x x}^{2}\right) u_{0}(\omega, x)<0 .\end{cases}
$$

Notice that

$$
\left\{V_{0}(\omega, x)>0\right\} \cap\left\{V_{0}(\omega, x)<0\right\}=\emptyset .
$$

Combining (6.15) and (6.16) yields

$$
\mathbb{P}\left\{\left|v_{x}(\omega, t, x)\right| \leq|v(\omega, t, x)|, \quad \forall(t, x) \in\left[0, \tau^{*}\right) \times \mathbb{T}\right\} \geq p+q .
$$

In view of $H^{1} \hookrightarrow L^{\infty},(6.3)$ and (6.17), we arrive at

$$
\begin{aligned}
& \mathbb{P}\left\{\left\|v_{x}(\omega, t)\right\|_{L^{\infty}} \leq\|v(\omega, t)\|_{L^{\infty}} \lesssim\|v(\omega, t)\|_{H^{1}}\right. \\
& \left.\quad=\left\|u_{0}\right\|_{H^{1}}, \quad \forall t \in\left[0, \tau^{*}\right)\right\} \geq p+q .
\end{aligned}
$$

Via (6.1), we obtain the desired estimate.

Proof of Theorem 2.4. Let $\left(u, \tau^{*}\right)$ be the maximal solution to (1.12). Then Lemma 6.2 implies that

$$
\mathbb{P}\left\{\|u\|_{W^{1, \infty}} \lesssim 2 \beta(\omega, t)\left\|u_{0}\right\|_{H^{1}}, \quad \forall t \in\left[0, \tau^{*}\right)\right\} \geq p+q
$$

It follows from (i) in Lemma 3.7 that $\sup _{t>0} \beta(\omega, t)<\infty \mathbb{P}-$ a.s. Then we can infer from (2.5) that $\mathbb{P}\left\{\tau^{*}=\infty\right\} \geq p+q$. That is to say, $\mathbb{P}\{u$ exists globally $\} \geq$ $p+q$. 


\subsection{Theorem 2.5: blow-up scenario}

Proof of Theorem 2.5. Recall (6.1). By (i) in Lemma 3.7, $A=A(\omega)=$ $\sup _{t>0} \beta(\omega, t)<\infty \mathbb{P}-$ a.s. Then we can first infer from $H^{1} \hookrightarrow L^{\infty}$ and (6.3) that for all $t>0$,

$$
\sup _{t>0}\|u\|_{L^{\infty}} \lesssim A\left\|u_{0}\right\|_{H^{1}}<\infty \mathbb{P}-\text { a.s. }
$$

which is (2.9). Now we prove (2.10). Let

$\Omega_{1}=\left\{\limsup _{t \rightarrow \tau^{*}}\|u(t)\|_{H^{s}}=\infty\right\} \quad$ and $\quad \Omega_{2}=\left\{\liminf _{t \rightarrow \tau^{*}}\left[\min _{x \in \mathbb{T}} u_{x}(t, x)\right]=-\infty\right\}$.

By the previously proven blow-up criterion in Theorem 2.1, we have that for a.e. $\omega \in \Omega_{2}, \omega \in \Omega_{1}$. Now we prove that for a.e. $\omega \in \Omega_{1}, \omega \in \Omega_{2}$. Suppose not. Then there is a positive random variable $K=K(\omega)<\infty$ almost surely such that

$$
u_{x}(\omega, t, x)>-K, \quad(t, x) \in\left[0, \tau^{*}(\omega)\right) \times \mathbb{T} \mathbb{P}-\text { a.s. }
$$

Using (6.12), (6.1) and integration by parts, we find that

$$
\begin{aligned}
\frac{\mathrm{d}}{\mathrm{d} t} \int_{\mathbb{T}} V^{2} \mathrm{~d} x & =2 \int_{\mathbb{T}} V\left[-\beta v V_{x}-2 \beta V v_{x}+\gamma V_{x}\right] \mathrm{d} x \\
& =-4 \beta \int_{\mathbb{T}} V^{2} v_{x} \mathrm{~d} x-2 \beta \int_{\mathbb{T}} V V_{x} v \mathrm{~d} x \\
& =-3 \beta \int_{\mathbb{T}} V^{2} v_{x} \mathrm{~d} x \leq 3 K \int_{\mathbb{T}} V^{2} \mathrm{~d} x, \quad t \in\left[0, \tau^{*}\right) \quad \mathbb{P}-\text { a.s. }
\end{aligned}
$$

which yields that

$$
\|V\|_{L^{2}} \lesssim \mathrm{e}^{3 K t}\|V(0)\|_{L^{2}}<\infty, \quad t \in\left[0, \tau^{*}\right) \quad \mathbb{P}-\text { a.s. }
$$

Combining the above estimate, (6.1) and $A(\omega)=\sup _{t>0} \beta(\omega, t)<\infty \mathbb{P}-$ a.s. (cf. (i) in Lemma 3.7), we have that

$$
\|u(t)\|_{H^{2}} \lesssim \beta(t) \mathrm{e}^{K t}\|u(0)\|_{H^{2}}<\infty, \quad t \in\left[0, \tau^{*}\right) \mathbb{P}-\text { a.s. }
$$

By the embedding $H^{2} \hookrightarrow W^{1, \infty}$ and the blow-up criterion in Theorem 2.1, almost surely we have that $\|u(t)\|_{H^{s}}$ can be extended beyond $\tau^{*}$. Therefore we obtain a contradiction and hence $\omega \in \Omega_{2}$. Therefore we obtain (2.10).

\subsection{Theorem 2.6: wave breaking and its probability}

The proof of Theorem 2.6 relies on certain properties of the solution $v$ to the problem (6.2).

Proposition 6.2. Let $\mathcal{S}=\left(\Omega, \mathcal{F}, \mathbb{P},\left\{\mathcal{F}_{t}\right\}_{t \geq 0}, W\right)$ be a fixed stochastic basis, let $b(t)$ satisfy Assumption 2.2, $c_{0}+\gamma=0, s>3$ and $u_{0}=u_{0}(x) \in H^{s}$ be an $H^{s}$ valued $\mathcal{F}_{0}$ measurable random variable with $\mathbb{E}\left\|u_{0}\right\|_{H^{s}}^{2}<\infty$. Let $\left(u, \tau^{*}\right)$ be the maximal solution to (1.11) with initial random variable $u_{0}$. Recall the process $\beta$ given in (6.1) and the constant $\lambda$ as in Eq. (2.11). Let $N=\frac{\lambda}{2}\left\|u_{0}\right\|_{H^{1}}^{2}<\infty$. Then for $v$, defined by (6.1), we have that

$$
M(\omega, t):=\min _{x \in \mathbb{T}}\left[v_{x}(\omega, t, x)\right]
$$


satisfies the following estimate almost surely:

$$
\frac{\mathrm{d}}{\mathrm{d} t} M(t) \leq \beta N-\beta \frac{1}{2} M^{2}(t) \text { a.e. on }\left(0, \tau^{*}\right) .
$$

Moreover, if $M(0)<-\sqrt{2 N}$ almost surely, then

$$
M(t) \leq-\sqrt{2 N}, \quad \forall t \in\left[0, \tau^{*}\right) \quad \mathbb{P}-\text { a.s. },
$$

and $M$ is non-increasing on $\left[0, \tau^{*}\right) \mathbb{P}-$ a.s.

Proof. For any $v \in H^{1}$, it is easy to see that, cf. [10],

$$
G_{\mathbb{T}} *\left(v^{2}+\frac{1}{2} v_{x}^{2}\right)(x) \geq \frac{1}{2} v^{2} .
$$

Using (1.9), (1.7), (6.4) and (6.1), we find for $c_{0}+\gamma=0$ that

$v_{t x}-\gamma v_{x x}+\beta v v_{x x}=\beta v^{2}-\beta \frac{1}{2} v_{x}^{2}-\beta G_{\mathbb{T}} *\left(v^{2}+\frac{1}{2} v_{x}^{2}\right), \quad t \in\left[0, \tau^{*}\right) \quad \mathbb{P}-$ a.s.

By Proposition 6.1, $v(\omega, t, x) \in C^{1}\left(\left[0, \tau^{*}\right) ; H^{s-1}\right)$ with $s>3$ almost surely. To apply Lemma 3.6 for each path, we recall $(6.18)$ and let $z(\omega, t)$ be a point where the infimum of $v_{x}$ is attained as in Lemma 3.6. Then for a.e. $\omega \in \Omega$, $v_{x x}(t, z(\omega, t))=0$. Moreover, Lemma 3.6 also implies that for a.e. $\omega \in \Omega$, the path of $M(\omega, t)$ is locally Lipschitz.

Then for almost all $t \in\left[0, \tau^{*}\right)$, evaluating (6.22) in $(t, z(t))$ with using Lemma 3.6 yields for a.e. $\omega \in \Omega$,

$$
\begin{aligned}
& \frac{\mathrm{d}}{\mathrm{d} t} M(t)=\beta v^{2}(t, z(t)) \\
& \quad-\beta \frac{1}{2} M^{2}(t)-\beta G_{\mathbb{T}} *\left(v^{2}+\frac{1}{2} v_{x}^{2}\right)(t, z(t)) \text { a.e. on }\left(0, \tau^{*}\right) .
\end{aligned}
$$

Since $\mathbb{E}\left\|u_{0}\right\|_{H^{s}}<\infty, N=\frac{\lambda}{2}\left\|u_{0}\right\|_{H^{1}}^{2}<\infty \mathbb{P}-$ a.s. Applying (6.21), (2.11) and (6.3) in the above equation gives that for a.e. $\omega \in \Omega$,

$$
\begin{aligned}
\frac{\mathrm{d}}{\mathrm{d} t} M(t) & \leq \beta \frac{1}{2} v^{2}(t, z(t))-\beta \frac{1}{2} M^{2}(t) \\
& \leq \beta \frac{\lambda}{2}\|v(t)\|_{H^{1}}^{2}-\beta \frac{1}{2} M^{2}(t) \\
& =\beta N-\beta \frac{1}{2} M^{2}(t) \text { a.e. on }\left(0, \tau^{*}\right),
\end{aligned}
$$

which is (6.19). In order to show (6.20), we define $\tau$ as

$$
\tau(\omega):=\inf \{t>0: M(\omega, t)>-\sqrt{2 N}\} \wedge \tau^{*} .
$$

If $M(0)<-\sqrt{2 N}$, then $\mathbb{P}\{\tau>0\}=1$. Now we only need to show that

$$
\mathbb{P}\left\{\tau(\omega)=\tau^{*}(\omega)\right\}=1 .
$$

Actually, failure of (6.24) would ensure the existence of a set $\Omega^{\prime} \subseteq \Omega$ such that $\mathbb{P}\left\{\Omega^{\prime}\right\}>0$ and $0<\tau\left(\omega^{\prime}\right)<\tau^{*}\left(\omega^{\prime}\right)$ for a.e. $\omega^{\prime} \in \Omega^{\prime}$. In view of the time continuity of $M$ (recall Lemma 3.6), we find that $M\left(\omega^{\prime}, \tau\left(\omega^{\prime}\right)\right)=-\sqrt{2 N}$. From 
(6.19) we have that $M\left(\omega^{\prime}, t\right)$ is non-increasing for $t \in\left[0, \tau\left(\omega^{\prime}\right)\right)$. Hence by the continuity of the path of $M\left(\omega^{\prime}, t\right)$ again, we see that $M\left(\omega^{\prime}, \tau\left(\omega^{\prime}\right)\right) \leq M(0)<$ $-\sqrt{2 N}$, which is a contradiction. Hence (6.24) is true and so is (6.20).

Proposition 6.3. Let all the conditions as in Proposition 6.2 hold true. Let $0<c<1$ and

$$
\Omega^{*}=\left\{\omega: \beta(t) \geq c \mathrm{e}^{-\frac{b^{*}}{2} t} \quad \text { for all } t\right\} .
$$

If $M(0)<-\frac{1}{2} \sqrt{\frac{\left(b^{*}\right)^{2}}{c^{2}}+8 N}-\frac{b^{*}}{2 c}$ almost surely, then for a.e. $\omega \in \Omega^{*}$,

$$
\tau^{*}(\omega)<\infty
$$

Proof. We rewrite (6.19) as

$$
\begin{aligned}
\frac{\mathrm{d}}{\mathrm{d} t} M(t) \leq & -\frac{\beta}{2}\left(1-\frac{2 N}{M^{2}(0)}\right) M^{2}(t) \\
& -\frac{\beta N}{M^{2}(0)} M^{2}(t)+\beta N \quad \text { a.e. on }\left(0, \tau^{*}\right) \quad \mathbb{P}-\text { a.s. }
\end{aligned}
$$

Due to Proposition 6.2, we have

$$
\begin{aligned}
\frac{\mathrm{d}}{\mathrm{d} t} M(t) & \leq-\frac{\beta(t)}{2}\left(1-\frac{2 N}{M^{2}(0)}\right) M^{2}(t)-\left(\frac{M^{2}(t)}{M^{2}(0)}-1\right) \beta(t) N \\
& \leq-\frac{\beta(t)}{2}\left(1-\frac{2 N}{M^{2}(0)}\right) M^{2}(t) \quad \text { a.e. on }\left(0, \tau^{*}\right) \quad \mathbb{P}-\text { a.s. }
\end{aligned}
$$

Since $M(t)$ is locally Lipschitz continuous in $t$ and satisfies $(6.20), \frac{1}{M(t)}$ is also locally Lipschitz continuous in $t$ almost surely. Therefore an integration leads to

$$
\frac{1}{M(t)}-\frac{1}{M(0)} \geq\left(1-\frac{2 N}{M^{2}(0)}\right) \int_{0}^{t} \frac{\beta\left(t^{\prime}\right)}{2} \mathrm{~d} t^{\prime}, \quad t \in\left(0, \tau^{*}\right) \mathbb{P}-\text { a.s. }
$$

which together with (6.20) means that for a.e. $\omega \in \Omega^{*}$,

$$
-\frac{1}{M(0)} \geq\left(\frac{1}{2}-\frac{N}{M^{2}(0)}\right) \int_{0}^{\tau^{*}} \beta(t) \mathrm{d} t \geq\left(\frac{1}{2}-\frac{N}{M^{2}(0)}\right)\left(\frac{2 c}{b^{*}}-\frac{2 c}{b^{*}} \mathrm{e}^{-\frac{b^{*}}{2} \tau^{*}}\right) .
$$

Recall that $M(0)<-\frac{1}{2} \sqrt{\frac{\left(b^{*}\right)^{2}}{c^{2}}+8 N}-\frac{b^{*}}{2 c}$ almost surely. We finally arrive at

$$
\left(\frac{1}{2}-\frac{N}{M^{2}(0)}\right) \frac{2 c}{b^{*}} \mathrm{e}^{-\frac{b^{*}}{2} \tau^{*}} \geq \frac{2 c}{b^{*}}\left(\frac{1}{2}-\frac{N}{M^{2}(0)}\right)+\frac{1}{M(0)}>0 \quad \text { a.e. on } \Omega^{*} .
$$

Therefore we have $\tau^{*}<\infty$ a.e. on $\Omega^{*}$.

Proof of Theorem 2.6. Proposition 6.3 implies that

$$
\mathbb{P}\left\{\tau^{*}<\infty\right\} \geq \mathbb{P}\left\{\beta(t) \geq c \mathrm{e}^{-\frac{b^{*}}{2} t} \text { for all } t\right\} .
$$

Since $b^{2}(t)<b^{*}$ for all $t>0$, we have

$$
\left\{\mathrm{e}^{\int_{0}^{t} b\left(t^{\prime}\right) \mathrm{d} W_{t^{\prime}}}>c \quad \text { for all } t\right\} \subseteq\left\{\beta(t) \geq c \mathrm{e}^{-\frac{b^{*}}{2} t} \quad \text { for all } t\right\} .
$$


Therefore we arrive at

$$
\mathbb{P}\left\{\tau^{*}<\infty\right\} \geq \mathbb{P}\left\{\mathrm{e}^{\int_{0}^{t} b\left(t^{\prime}\right) \mathrm{d} W_{t^{\prime}}}>c \text { for all } t\right\}>0,
$$

which gives the desired estimate in Theorem 2.6.

\subsection{Theorem 2.7: wave breaking rate}

As the last contribution of the paper, we prove Theorem 2.7, which provides a precise bound on the wave breaking rate.

Proof of Theorem 2.7. Recalling (6.23), we have that almost surely

$$
-\beta\left\|G_{\mathbb{T}} *\left(v^{2}+\frac{1}{2} v_{x}^{2}\right)\right\|_{L^{\infty}} \leq \frac{\mathrm{d}}{\mathrm{d} t} M(t)+\beta \frac{1}{2} M^{2}(t) \leq \beta\|v\|_{L^{\infty}}^{2} \quad \text { a.e. on }\left(0, \tau^{*}\right) \text {. }
$$

Using $\left\|G_{\mathbb{T}}\right\|_{L^{\infty}}<\infty$ and (6.3), we have

$$
\left\|G_{\mathbb{T}^{*}} *\left(v^{2}+\frac{1}{2} v_{x}^{2}\right)\right\|_{L^{\infty}} \lesssim\left\|v^{2}+\frac{1}{2} v_{x}^{2}\right\|_{L^{1}} \lesssim\|v\|_{H^{1}}^{2}=\left\|u_{0}\right\|_{H^{1}}^{2}
$$

Therefore there is a constant $C>0$ such that

$$
-C \beta\left\|u_{0}\right\|_{H^{1}}^{2} \leq \frac{\mathrm{d}}{\mathrm{d} t} M(t)+\beta \frac{1}{2} M^{2}(t) \leq C \beta\left\|u_{0}\right\|_{H^{1}}^{2} \quad \text { a.e. on }\left(0, \tau^{*}\right) .
$$

Let $\varepsilon \in\left(0, \frac{1}{2}\right)$ and $K=C\left\|u_{0}\right\|_{H^{1}}^{2}$. Since $\liminf _{t \rightarrow \tau^{*}} M(t)=-\infty$ a.e. on $\left\{\tau^{*}<\infty\right\}$ and $K<\infty$ almost surely (cf. Theorem 2.5), for a.e. $\omega \in \Omega$, there is some $t_{0}=t_{0}(\omega, \varepsilon) \in\left(0, \tau^{*}\right)$ with $M\left(t_{0}\right)<0$ and $M^{2}\left(t_{0}\right)>\frac{K}{\varepsilon}$. Similar to the proof of (6.20), we have that for a.e. $\omega \in\left\{\tau^{*}<\infty\right\}$,

$$
M^{2}(t)>\frac{K}{\varepsilon}, \quad t \in\left[t_{0}, \tau^{*}\right) .
$$

A combination of (6.25) and (6.26) enables us to infer that for a.e. $\omega \in\left\{\tau^{*}<\right.$ $\infty\}$,

$$
\beta \frac{K}{M^{2}(t)}+\frac{\beta}{2}>-\frac{\frac{\mathrm{d}}{\mathrm{d} t} M(t)}{M^{2}(t)}>-\beta \frac{K}{M^{2}(t)}+\frac{\beta}{2} \quad \text { a.e. on }\left(t_{0}, \tau^{*}\right) .
$$

Since for a.e. $\omega \in\left\{\tau^{*}<\infty\right\}$, the path of $M$ is locally Lipschitz in $t$ and satisfies (6.26), $\frac{1}{M}$ is also locally Lipschitz.

Then we integrate the above estimate on $\left(t, \tau^{*}\right)$ to derive that for a.e. $\omega \in\left\{\tau^{*}<\infty\right\}$

$$
\left(\frac{1}{2}+\varepsilon\right) \int_{t}^{\tau^{*}} \beta\left(t^{\prime}\right) \mathrm{d} t^{\prime} \geq-\frac{1}{M(t)} \geq\left(\frac{1}{2}-\varepsilon\right) \int_{t}^{\tau^{*}} \beta\left(t^{\prime}\right) \mathrm{d} t^{\prime}, \quad t_{0}<t<\tau^{*} .
$$

Therefore we can infer from (6.1) and (6.18) that for a.e. $\omega \in\left\{\tau^{*}<\infty\right\}$,

$$
\frac{1}{\frac{1}{2}+\varepsilon} \leq-\min _{x \in \mathbb{T}}\left[u_{x}(\omega, t, x)\right] \beta^{-1}(t) \int_{t}^{\tau^{*}} \beta\left(t^{\prime}\right) \mathrm{d} t^{\prime} \leq \frac{1}{\frac{1}{2}-\varepsilon}, \quad t_{0}<t<\tau^{*} .
$$

Since $\varepsilon \in\left(0, \frac{1}{2}\right)$ is arbitrary, we obtain that for $\beta(\omega, t)=\mathrm{e}_{0}^{t} b\left(t^{\prime}\right) \mathrm{d} W_{t^{\prime}}-\int_{0}^{t} \frac{b^{2}\left(t^{\prime}\right)}{2} \mathrm{~d} t^{\prime}$,

$$
\lim _{t \rightarrow \tau^{*}}\left(\min _{x \in \mathbb{T}}\left[u_{x}(t, x)\right] \int_{t}^{\tau^{*}} \beta\left(t^{\prime}\right) \mathrm{d} t^{\prime}\right)=-2 \beta\left(\tau^{*}\right) \quad \text { a.e. on } \quad\left\{\tau^{*}<\infty\right\}
$$


which completes the proof.

\section{Acknowledgements}

The authors would like to express their great gratitude to the anonymous referees for their important suggestions, which have led to a significant improvement of this paper. Hao Tang is deeply indebted to Professor Feng-Yu Wang for his insightful suggestions on the Lyapunov-type condition on the noise.

Funding Open Access funding enabled and organized by Projekt DEAL.

Open Access. This article is licensed under a Creative Commons Attribution 4.0 International License, which permits use, sharing, adaptation, distribution and reproduction in any medium or format, as long as you give appropriate credit to the original author(s) and the source, provide a link to the Creative Commons licence, and indicate if changes were made. The images or other third party material in this article are included in the article's Creative Commons licence, unless indicated otherwise in a credit line to the material. If material is not included in the article's Creative Commons licence and your intended use is not permitted by statutory regulation or exceeds the permitted use, you will need to obtain permission directly from the copyright holder. To view a copy of this licence, visit http://creativecommons. org/licenses/by/4.0/.

Publisher's Note Springer Nature remains neutral with regard to jurisdictional claims in published maps and institutional affiliations.

\section{References}

[1] Bensoussan, A.: Stochastic Navier-Stokes equations. Acta Appl. Math. 38(3), 267-304 (1995)

[2] Breit, D., Feireisl, E., Hofmanová, M.: Local strong solutions to the stochastic compressible Navier-Stokes system. Commun. Partial Differ. Equ. 43(2), 313$345(2018)$

[3] Breit, D., Feireisl, E., Hofmanová, M.: Stochastically Forced Compressible Fluid Flows. De Gruyter Series in Applied and Numerical Mathematics, vol. 3. De Gruyter, Berlin (2018)

[4] Breit, D., Hofmanová, M.: Stochastic Navier-Stokes equations for compressible fluids. Indiana Univ. Math. J. 65(4), 1183-1250 (2016)

[5] Bressan, A., Constantin, A.: Global conservative solutions of the Camassa-Holm equation. Arch. Ration. Mech. Anal. 183(2), 215-239 (2007)

[6] Bressan, A., Constantin, A.: Global dissipative solutions of the Camassa-Holm equation. Anal. Appl. (Singap.) 5(1), 1-27 (2007) 
[7] Brzeźniak, Z., Capiński, M., Flandoli, F.: Stochastic partial differential equations and turbulence. Math. Models Methods Appl. Sci. 1(1), 41-59 (1991)

[8] Chen, Y., Gao, H.: Well-posedness and large deviations of the stochastic modified Camassa-Holm equation. Potential Anal. 45(2), 331-354 (2016)

[9] Chen, Y., Gao, H., Guo, B.: Well-posedness for stochastic Camassa-Holm equation. J. Differ. Equ. 253(8), 2353-2379 (2012)

[10] Constantin, A.: On the blow-up of solutions of a periodic shallow water equation. J. Nonlinear Sci. 10(3), 391-399 (2000)

[11] Constantin, A.: The trajectories of particles in Stokes waves. Invent. Math. 166(3), 523-535 (2006)

[12] Constantin, A., Escher, J.: Wave breaking for nonlinear nonlocal shallow water equations. Acta Math. 181(2), 229-243 (1998)

[13] Constantin, A., Escher, J.: Well-posedness, global existence, and blowup phenomena for a periodic quasi-linear hyperbolic equation. Commun. Pure Appl. Math. 51(5), 475-504 (1998)

[14] Constantin, A., Escher, J.: Particle trajectories in solitary water waves. Bull. Am. Math. Soc. (N.S.) 44(3), 423-431 (2007)

[15] Constantin, A., Escher, J.: Analyticity of periodic traveling free surface water waves with vorticity. Ann. Math. (2) 173(1), 559-568 (2011)

[16] Crisan, D., Flandoli, F., Holm, D.D.: Solution properties of a 3D stochastic Euler fluid equation. J. Nonlinear Sci. 29(3), 813-870 (2019)

[17] Crisan, D., Holm, D.D.: Wave breaking for the stochastic Camassa-Holm equation. Phys. D 376(377), 138-143 (2018)

[18] Da Prato, G., Zabczyk, J.: Stochastic Equations in Infinite Dimensions. Encyclopedia of Mathematics and its Applications, vol. 152, 2nd edn. Cambridge University Press, Cambridge (2014)

[19] Debussche, A., Glatt-Holtz, N.E., Temam, R.: Local martingale and pathwise solutions for an abstract fluids model. Phys. D 240(14-15), 1123-1144 (2011)

[20] Dullin, H.R., Gottwald, G.A., Holm, D.D.: An integrable shallow water equation with linear and nonlinear dispersion. Phys. Rev. Lett. 87, 194501 (2001)

[21] W.E. Stochastic hydrodynamics. In: Current Developments in Mathematics, 2000, pp. 109-147. Int. Press, Somerville, MA (2001)

[22] Fedrizzi, E., Flandoli, F.: Noise prevents singularities in linear transport equations. J. Funct. Anal. 264(6), 1329-1354 (2013)

[23] Flandoli, F.: Random perturbation of PDEs and fluid dynamic models, volume 2015 of Lecture Notes in Mathematics. Springer, Heidelberg, 2011. Lectures from the 40th Probability Summer School held in Saint-Flour, 2010, École d'Été de Probabilités de Saint-Flour. [Saint-Flour Probability Summer School] 
[24] Flandoli, F., Gubinelli, M., Priola, E.: Well-posedness of the transport equation by stochastic perturbation. Invent. Math. 180(1), 1-53 (2010)

[25] Flandoli, F., Gubinelli, M., Priola, E.: Full well-posedness of point vortex dynamics corresponding to stochastic 2D Euler equations. Stoch. Process. Appl. 121(7), 1445-1463 (2011)

[26] Gawarecki, L., Mandrekar, V.: Stochastic differential equations in infinite dimensions with applications to stochastic partial differential equations. Probability and its Applications (New YorK). Springer, Heidelberg (2011)

[27] Gess, B., Souganidis, P.E.: Long-time behavior, invariant measures, and regularizing effects for stochastic scalar conservation laws. Commun. Pure Appl. Math. 70(8), 1562-1597 (2017)

[28] Glatt-Holtz, N., Ziane, M.: Strong pathwise solutions of the stochastic NavierStokes system. Adv. Differ. Equ. 14(5-6), 567-600 (2009)

[29] Glatt-Holtz, N.E., Vicol, V.C.: Local and global existence of smooth solutions for the stochastic Euler equations with multiplicative noise. Ann. Probab. 42(1), 80-145 (2014)

[30] Gyöngy, I., Krylov, N.: Existence of strong solutions for Itô's stochastic equations via approximations. Probab. Theory Relat. Fields 105(2), 143-158 (1996)

[31] Holden, H., Raynaud, X.: Global conservative solutions of the Camassa-Holm equation - a Lagrangian point of view. Commun. Partial Differ. Equ. 32(10-12), 1511-1549 (2007)

[32] Holden, H., Raynaud, X.: Dissipative solutions for the Camassa-Holm equation. Discrete Contin. Dyn. Syst. 24(4), 1047-1112 (2009)

[33] Kallianpur, G., Xiong, J.: Stochastic differential equations in infinitedimensional spaces. 26:vi+342. Expanded version of the lectures delivered as part of the 1993 Barrett Lectures at the University of Tennessee, Knoxville, TN, March 25-27, 1993. With a foreword by Balram S, Rajput and Jan Rosinski (1995)

[34] Kato, T., Ponce, G.: Commutator estimates and the Euler and Navier-Stokes equations. Commun. Pure Appl. Math. 41(7), 891-907 (1988)

[35] Kenig, C.E., Ponce, G., Vega, L.: Well-posedness and scattering results for the generalized Korteweg-de Vries equation via the contraction principle. Commun. Pure Appl. Math. 46(4), 527-620 (1993)

[36] Kim, J.U.: On the Cauchy problem for the transport equation with random noise. J. Funct. Anal. 259(12), 3328-3359 (2010)

[37] Kröker, I., Rohde, C.: Finite volume schemes for hyperbolic balance laws with multiplicative noise. Appl. Numer. Math. 62(4), 441-456 (2012)

[38] Krylov, N.V., Rozovskiǔ, B.L.: Stochastic evolution equations. In: Current Problems in Mathematics, Vol. 14 (Russian), pp. 71-147, 256. Akad. Nauk SSSR, Vsesoyuz. Inst. Nauchn. i Tekhn. Informatsii, Moscow (1979) 
[39] Kuksin, S., Shirikyan, A.: Mathematics of Two-Dimensional Turbulence. Cambridge Tracts in Mathematics, vol. 194. Cambridge University Press, Cambridge (2012)

[40] Lenells, J., Wunsch, M.: On the weakly dissipative Camassa-Holm, DegasperisProcesi, and Novikov equations. J. Differ. Equ. 255(3), 441-448 (2013)

[41] Liu, Y.: Global existence and blow-up solutions for a nonlinear shallow water equation. Math. Ann. 335(3), 717-735 (2006)

[42] Lv, W., He, P., Wang, Q.: Well-posedness and blow-up solution for the stochastic Dullin-Gottwald-Holm equation. J. Math. Phys. 60(8), 083513, 10 (2019)

[43] McKean, H.P.: Breakdown of a shallow water equation. Asian J. Math. 2(4), 867-874 (1998). (Mikio Sato: a great Japanese mathematician of the twentieth century)

[44] Prévôt, C., Röckner, M.: A concise course on stochastic partial differential equations. Lecture Notes in Mathematics, vol. 1905. Springer, Berlin (2007)

[45] Ren, P., Tang, H., Wang, F.-Y.: Distribution-path dependent nonlinear SPDEs with application to stochastic transport type equations. arXiv:2007.09188 (2020)

[46] Röckner, M., Zhu, R., Zhu, X.: Local existence and non-explosion of solutions for stochastic fractional partial differential equations driven by multiplicative noise. Stoch. Process. Appl. 124(5), 1974-2002 (2014)

[47] Rohde, C., Tang, H.: On a stochastic Camassa-Holm type equation with higher order nonlinearities. J. Dyn. Differ. Equ. https://doi.org/10.1007/ s10884-020-09872-1

[48] Tang, H.: On the pathwise solutions to the Camassa-Holm equation with multiplicative noise. SIAM J. Math. Anal. 50(1), 1322-1366 (2018)

[49] Tang, H.: Noise effects on dependence on initial data and blow-up for stochastic Euler-Poincaré equations. arXiv:2002.08719 (2020)

[50] Tang, H., Zhao, Y., Liu, Z.: A note on the solution map for the periodic CamassaHolm equation. Appl. Anal. 93(8), 1745-1760 (2014)

[51] Whitham, G.B.: Linear and Nonlinear Waves. Pure and Applied Mathematics (New YorK). Wiley, New York (1999). Reprint of the 1974 original, A WileyInterscience Publication

[52] Wu, S., Yin, Z.: Blow-up and decay of the solution of the weakly dissipative Degasperis-Procesi equation. SIAM J. Math. Anal. 40(2), 475-490 (2008)

[53] Wu, S., Yin, Z.: Global existence and blow-up phenomena for the weakly dissipative Camassa-Holm equation. J. Differ. Equ. 246(11), 4309-4321 (2009) 
Christian Rohde and Hao Tang

Institut für Angewandte Analysis und Numerische Simulation

Universität Stuttgart

Pfaffenwaldring 57

70569 Stuttgart

Germany

e-mail: Hao.Tang@mathematik.uni-stuttgart.de

Christian Rohde

e-mail: christian.rohde@mathematik.uni-stuttgart.de

Received: 11 March 2020.

Accepted: 15 October 2020. 